\title{
A high-fidelity wave-to-wire simulation platform for wave energy converters: Coupled numerical wave tank and power take-off models
}

\author{
Markel Penalba*, Josh Davidson, Christian Windt, John V. Ringwood \\ Centre for Ocean Energy Research, Maynooth University, Maynooth, Co. Kildare, Ireland
}

H I G H L I G H T S

- A novel high-fidelity simulation platform is presented coupling CFD and a PTO model.

- CFD-based approaches are reinforced for applications where high-fidelity is vital.

- Significant overestimation is observed for excessively simplified PTO models.

- Minor inaccuracies in a conversion stage can significantly affect the power estimate.

\section{A R T I C L E I N F O}

\section{Keywords:}

Wave energy

Wave-to-wire modelling

CFD

Numerical Wave Tank

Power take-off

High-fidelity

\begin{abstract}
A B S T R A C T
Performing rigorous technical and commercial assessment of wave energy converters (WECs) numerically, before engaging in expensive wave tank and open ocean tests, is vital for the economically successful development of prototypes. To that end, this paper presents a high-fidelity wave-to-wire simulation platform (the HiFiWEC), where a Computational Fluid Dynamics (CFD)-based numerical wave tank is coupled to a high-fidelity power take-off (PTO) model, which enables assessment of WEC performance with greater accuracy than with previous wave-to-wire approaches. A test case, simulating the performance of a heaving point absorber type WEC in realistic conditions, is presented and compared against traditional lower fidelity modelling methods. The WEC response is evaluated with a number of different approaches, including different techniques to model hydrodynamic wave-structure interactions and the power take-off system, and the benefits of the HiFiWEC are highlighted. The results highlight that excessive simplifications in the modelling of the PTO system can lead to significant overestimation in generated energy output, with relative deviations $(\epsilon)$ of up to $150 \%$ compared to the HiFiWEC. In addition, uncertainty in viscous drag parameters added to hydrodynamic models based on boundary element method solvers, reinforce the necessity of CFD-based models for applications where highfidelity is essential. Finally, it is demonstrated that minor/insignificant inaccuracies in the hydrodynamic model $(\epsilon=0.5 \%)$ can result in significant differences in the estimation of the final energy generation $(\epsilon=7 \%)$, highlighting the need for a coupled high-fidelity platform.
\end{abstract}

\section{Introduction}

Clean energy technologies are fundamental to the development of a low-carbon environment, to mitigate the effects of human-induced climate change. Currently, about $20 \%$ of mankind's energy consumption is supplied by renewable energy sources, such as hydropower, wind or solar energy [1]. However, in the coming decades, a much larger share of the energy supply must be provided by renewables, requiring the contribution of additional renewable energy sources to the mix. Ocean waves present a tremendous untapped energy resource, about $32,000 \mathrm{TWh}$ /year according to [2], which could make a substantial contribution to the future supply of clean energy. However, the ocean is an extremely harsh environment, making the extraction of wave energy complex and expensive [3]. Due to this complexity, over 200 different prototypes have been suggested to harvest wave energy [4], but none have yet demonstrated commercial viability.

The development of wave energy converters (WECs) is a slow, risky and expensive process. The evolution, from the initial idea through to the final commercially competitive device, requires a number of distinct development stages. Assessing the commercial and economical ability of the WEC, at different stages of its development path, can be quantified using technology readiness levels (TRLs) and technology

\footnotetext{
* Corresponding author.

E-mail addresses: markel.penalbaretes.2015@mumail.ie (M. Penalba), Josh.Davidson@mu.ie (J. Davidson), christian.windt.2017@mumail.ie (C. Windt), john.ringwood@mu.ie (J.V. Ringwood).
} 


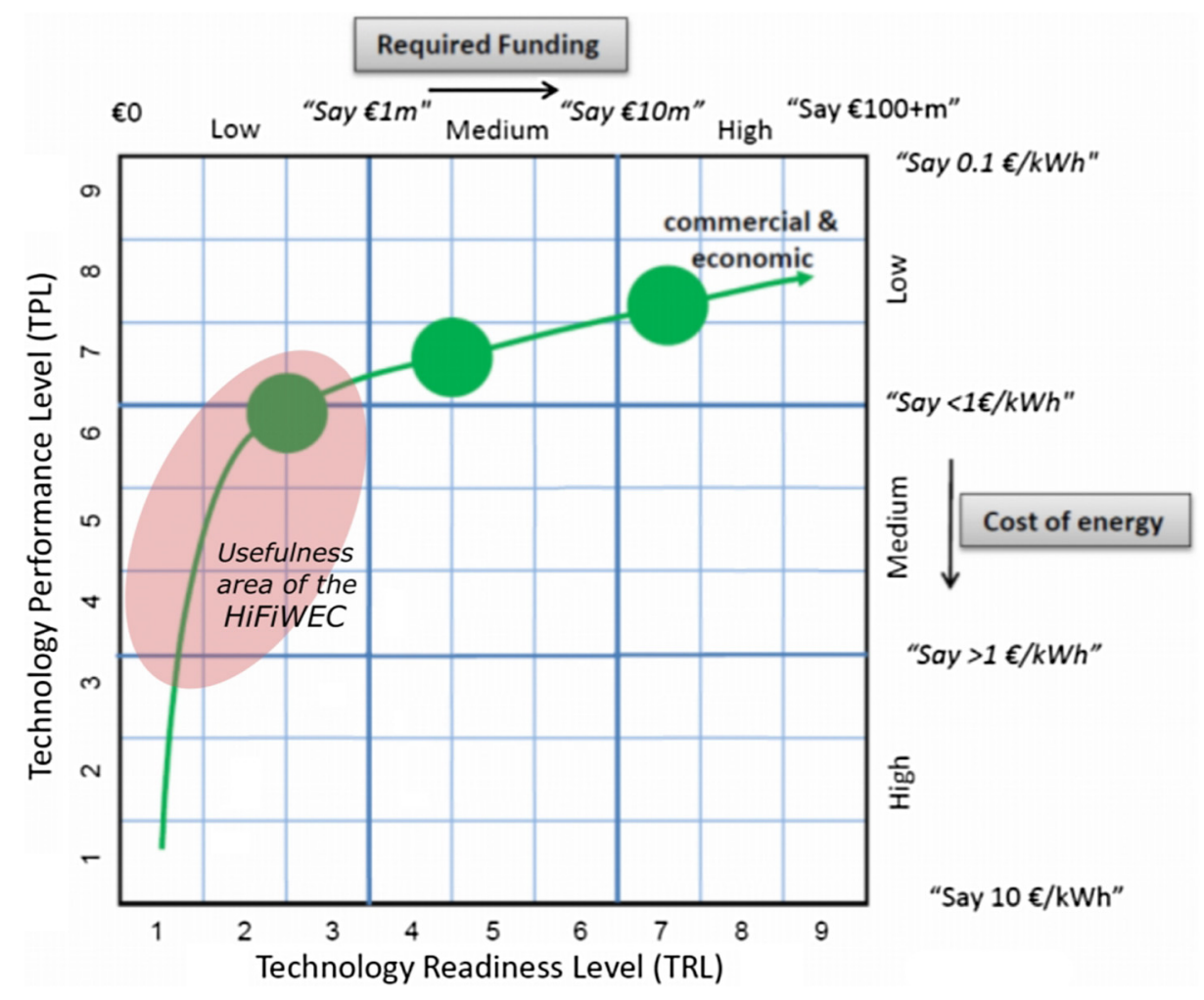

Fig. 1. TRL and TPL matrix with the ideal development trajectory and the applicability area of the HiFiWEC, adapted from [5].

performance levels (TPLs), respectively, as described in [5]. The ideal development trajectory, suggested in [5], delays the expensive prototype demonstration at higher TRLs, until reaching a high level of confidence on the concept, by first traversing the TPL scale at lower, less costly, TRLs, as illustrated in Fig. 1.

To evaluate the performance of a WEC at low TRLs, simulation models are required with increasing accuracy for increasing TPLs: the higher the TPL, the higher the level of modelling accuracy is needed to realistically assess the increase in WEC performance, and correspondingly the higher the accepted computational cost for the simulations. Therefore, to rigorously assess and optimise a WEC at low TRLs, before undertaking the critically expensive demonstration stages at higher TRLs, high-fidelity simulation models are essential to gain confidence in the expected WEC performance. These high-fidelity models must be able to evaluate the holistic performance of WECs, including the wavestructure interaction (WSI) and the power take-off (PTO) drivetrain. Such holistic models are typically termed wave-to-wire (W2W) models and are reviewed in [6].

Separate high-fidelity models for hydrodynamic WSIs and different PTO systems have been suggested in the literature. Computational Fluid Dynamics (CFD) are used to solve WSIs for various WEC types, such as point absorbers $[7,8]$, oscillating wave surge converters $[9,10]$ or oscillating water columns [11,12] (see a full review in [13]). However, W2W models that incorporate CFD to model hydrodynamic WSIs always use excessively simplified PTO representations, i.e. linear springdamper systems [13], resulting in highly unbalanced W2W models with an unjustifiable computational cost.

Similarly, high-fidelity models for different PTO systems have been suggested in the literature. These high-fidelity PTO models are, in general, coupled to relatively simple hydrodynamic models, mostly using a linear potential flow model based on Cummins' equation, which is sometimes extended with a quadratic viscous model, in the most complicated cases. Examples of W2W models with relatively high-fidelity PTO models can be found in the literature for different PTO system, e.g. air turbines [14-18], hydraulic PTO systems [19-26], mechanical transmission systems coupled to rotational electric generators $[27,28]$ or linear generators [29-31] (see a full review of W2W models in [6]).

However, to the authors' knowledge, no published model offers the possibility to evaluate the performance of WECs in a holistic numerical test bed, including sufficient fidelity of both the hydrodynamic and PTO models simultaneously. The only parsimonious model that evaluates the holistic performance of a WEC is presented in [32] (referred to as the NLBEMW2W model in the following sections), for which the hydrodynamic WSIs are solved via a partially nonlinear model based on boundary element method (BEM) codes, such as NEMOH [33].

To fill this gap, the present paper presents a novel holistic highfidelity W2W simulation platform, the HiFiWEC, which is the first attempt to couple a high-fidelity CFD-based numerical wave tank (CNWT) to a high-fidelity PTO model. Hence, the HiFiWEC offers a high-fidelity simulation model for medium-high TPLs and low-medium TRLs, as shown in Fig. 1.

The HiFiWEC can be particularly useful:

- as a benchmark to validate lower fidelity or computationally more efficient mathematical models [34],

- for system identification purposes, identifying the viscous drag coefficient [35] or representative/parametric models [36,37] under realistic operational conditions,

- to evaluate the efficacy of control strategies in realistic conditions $[38,39]$.

While these applications would traditionally have required physical wave tank experiments, the HiFiWEC offers some advantages compared to its physical counterpart. The HiFiWEC can eliminate undesired influences of measurement equipment and the test environment, e.g. the unrepeatability of experiments [40], reflections from tank walls [41] and friction from device restraints at small scale [42], as well as to evaluate devices at full scale [10]. Also, the difficulty in evaluating the performance of full-scale PTO systems and their impact on 
hydrodynamic WSI in wave tank experiments [43], is overcome in the HiFiWEC. However, it should be noted that the validation of the $\mathrm{Hi}$ FiWEC is important to gain confidence in the numerical platform, as described in Sections 2.1 and 2.2.

In contrast, it should also be noted that, due to the high computational cost, this high-fidelity platform is not useful for:

- power assessment across a wide range of sea states,

- model based control, or

- any kind of optimization purpose.

The remainder of the paper is as follows: Section 2 presents the high-fidelity platform, including the CNWT model, the PTO model and the coupling between both. A case study demonstration of the HiFiWEC is then presented, with the test cases described in Section 3 and results presented in Section 4. These results are discussed in Section 5 and a number of conclusions are then drawn in Section 6.

\section{High-fidelity simulation platform}

The high-fidelity simulation platform, the HiFiWEC, presented in this paper, is formed by coupling two high-fidelity models: a CNWT model that solves the fully nonlinear hydrodynamic WSI and a hydraulic PTO model that includes all the relevant dynamics, losses and constraints of hydraulic and electric subsystems. The novel contribution presented in the present study is the coupling of these models and the resulting analysis. Fig. 2 illustrates the coupling between the CNWT and PTO models, where the simulation characteristics, such as the WEC device and input waves $(\eta)$, are input to the CNWT and the final output of the HiFiWEC is the generated electric power $\left(P_{\text {elec }}\right)$. Since the CNWT and PTO models are implemented in different software environments, and use different time-integration solvers, the platform requires a bespoke communication channel to couple the two models, which is described in more detail in Section 2.3.

The validation of the HiFiWEC, as a whole, is very challenging, due to the large number of components included in wave-to-wire models, and the cost associated with building a physical model that includes all these components. In addition, results from small-scale PTO systems are unrepresentative for the validation of PTO models [24,44], which suggests that small-scale wave tank experiments cannot be used for the validation of the HiFiWEC. Therefore, the validation of the HiFiWEC is accomplished by validating the CNWT and the PTO model separately. Both the CNWT and the PTO model have been verified and validated in previous studies, detailed in Sections 2.1 and 2.2, respectively.

\subsection{CFD-based numerical wave tank}

Numerical wave tanks have been used for many decades in ocean engineering to analyse WSI [45]. The fluid dynamics problem traditionally relies on many linearising assumptions such as inviscid, irrotational and incompressible fluids to allow a computationally tractable solution, whereby NWTs were typically implemented based on the linear theory of the velocity potential and boundary integral equations. While these linearising assumptions provide acceptable accuracy for some offshore applications, WECs are designed to resonate with the incident waves, resulting in large amplitude motions which challenge

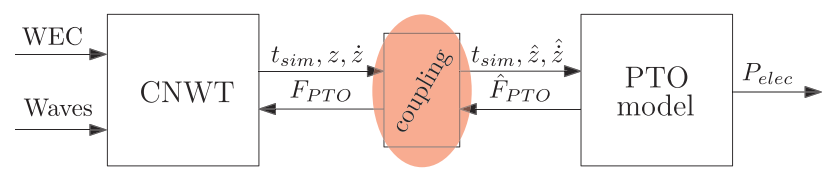

Fig. 2. Scheme of the high-fidelity simulation platform illustrating the coupling between the CNWT and the PTO model. Due to differences between the timeintegration solvers, interpolation of variables is inevitable, which are illustrated with a hat. linearisation, due to viscous drag, flow separation, vortex shedding and other nonlinear hydrodynamic effects. However, with the continuing increase in computer power, NWTs for WEC experiments can nowadays be implemented using CFD (see review [13]), which provides rigorous nonlinear treatment of the fluid dynamics problem [46].

The CNWT model in the HiFiWEC is based on the open-source CFD software, OpenFOAM [47]. The OpenFOAM based CNWT, captures relevant hydrodynamic non-linearities when simulating WEC operation, by numerically solving the incompressible Reynolds Averaged Navier-Stokes (RANS) equations using a cell-centred finite volume method. The RANS equations describe the conservation of mass and momentum, respectively given as:

$\nabla \cdot \mathbf{u}=0$

$\frac{\partial(\rho \mathbf{u})}{\partial t}+\nabla \cdot(\rho \mathbf{u u})=-\nabla p+\nabla \cdot \mathbf{T}+\rho \mathbf{f}_{b}$

where $t$ is time, $\mathbf{u}$ the fluid velocity, $p$ the fluid pressure, $\rho$ the fluid density, $\mathbf{T}$ the stress tensor and $\mathbf{f}_{b}$ the external forces such as gravity. Turbulence is included in the CNWT using a k-omegaSST turbulence model, which is the most commonly employed turbulence model for WEC applications [13], and utilises the kqRWallFunction for turbulence effects on the WEC boundary. The CNWT uses the interDyMFOAM solver to iteratively solve the RANS equations using the PIMPLE algorithm [48], and employs the Volume of Fluid (VoF) method to account for the two fluid phases in the NWT (air and water) and capture the free surface interface.

The interDyMFOAM solver allows dynamic mesh deformation to accomodate the motion of the WEC within the numerical domain. The WEC motion, due to input waves and PTO forces, is calculated using the sixDoFRigidBodyMotion solver [49]. A range of numerical wave makers are available in OpenFOAM to generate and absorb waves [50]. For the present case study the relaxation method is employed, via the $w a$ ves2Foam toolbbox [51], where target solutions for surface elevation and the velocity field are relaxed into the computational domain.

Full details of the CNWT implementation can be found in [52]. The setup of the CNWT has first been verified comparing the performance of a WEC simulated in the CNWT against results of a BEM model $[36,39,53]$, and then validated against physical experiments of a 1:10 scale WEC in [54].

\subsection{Power take-off model}

The PTO model included in this platform is implemented in MATLAB $^{\circledast}$ and considers a PTO system comprising a hydraulic transmission system and an electrical generator. Indeed, the platform can simulate the two different hydraulic transmission systems commonly used in wave energy [55]: constant- and variable-pressure configurations. However, for the sake of brevity, only the variable-pressure hydraulic system configuration is considered in this paper.

Hence, the hydraulic system implemented for this paper includes a hydraulic cylinder, a low-pressure accumulator, relief valves and a variable-displacement hydraulic motor coupled to a squirrel cage induction generator, as illustrated in Fig. 3.

The mathematical model for the hydraulic cylinder includes endstop constraints, friction losses, and compressibility and inertia effects, providing the final PTO force as follows,

$F_{P T O}=A_{p} \Delta p+F_{\text {fric }}+F_{I}$

where $A_{p}$ is the piston area, $\Delta p$ the pressure difference between the different cylinder chambers, $F_{\text {fric }}$ the friction force and $F_{I}$ the inertia force. Pressure dynamics in cylinder chambers, including compressibility effects, are modelled using the continuity equation,

$\dot{p}=\frac{\beta_{\text {eff }}}{V+A_{p} x_{p}}\left(Q-\dot{x}_{p} A_{p}\right)$ 


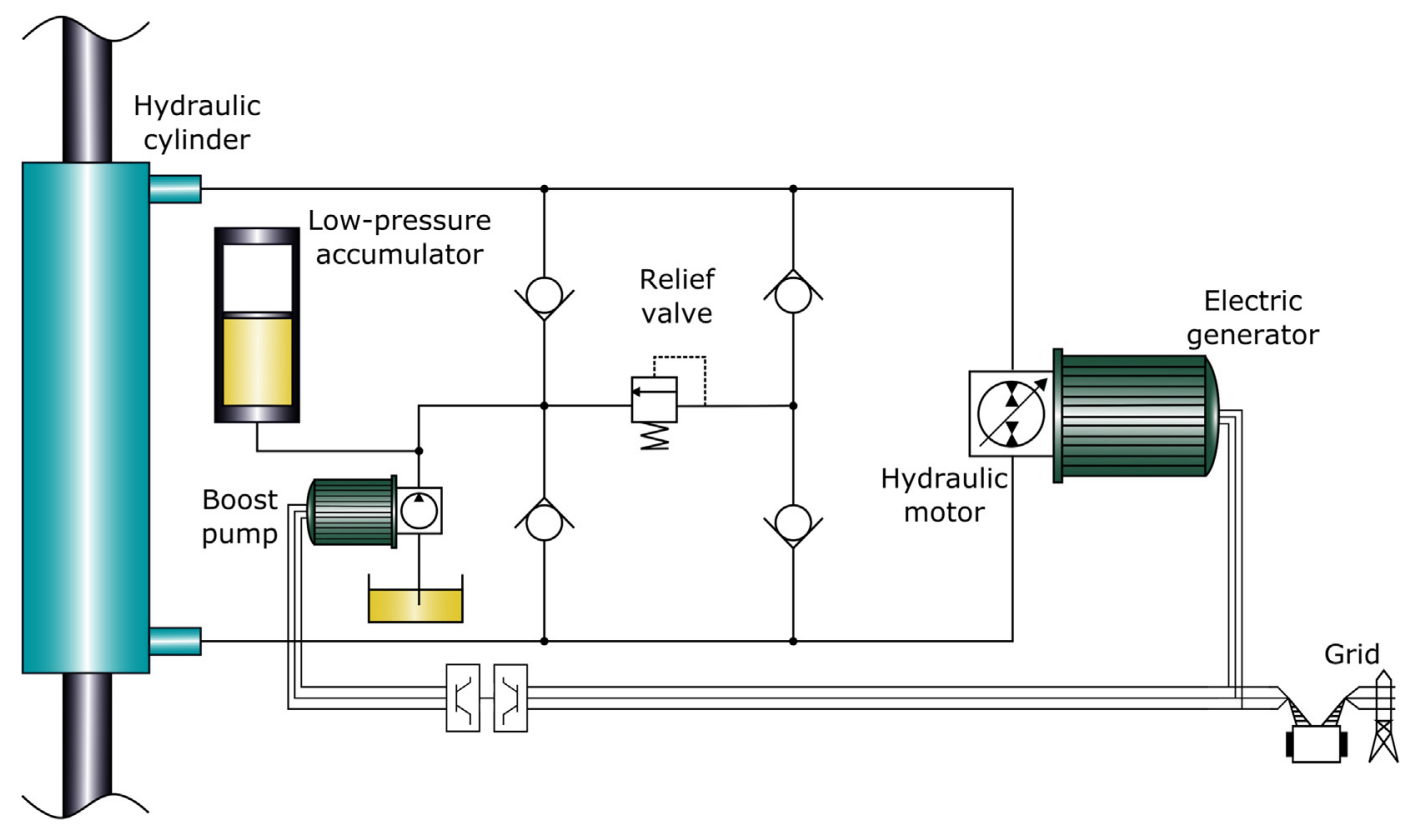

Fig. 3. Diagram of the PTO system implemented in the platform, including all the required components.

where $\beta_{\text {eff }}$ is the effective bulk modulus, $V$ the minimum volume in the cylinder chamber, $x_{p}$ and $\dot{x}_{p}$ the piston position and velocity, respectively, and $Q$ the flow entering or exiting the cylinder chamber.

Friction in the cylinder is modelled using the Stribeck model, which includes viscous, Coulomb and static friction [56], and the inertia force considers the inertial contributions of the cylinder piston $\left(M_{p}\right), \operatorname{rod}\left(M_{r}\right)$ and oil $\left(M_{o i l}\right)$ due to the piston acceleration,

$F_{\text {fric }}=\sigma_{v} \dot{x}_{p}+\operatorname{sign}\left(\dot{x}_{p}\right)\left[F_{c}+F_{s t} \exp \left(-\frac{\left|\dot{x}_{p}\right|}{c_{s t}}\right)\right]$

$F_{I}=\ddot{x}_{p}\left(M_{p}+M_{r}+M_{\text {oil }}\right)$

where $\sigma_{v}$ is the viscous coefficient, $F_{c}$ the Coulomb friction force, $F_{s t}$ the static friction force, $c_{s t}$ the characteristic velocity of the Stribeck curve, and $\ddot{x}_{p}$ the piston acceleration.

Relief valves are passive check-valves that open only if pressure in the hoses exceeds the maximum pressure allowed, and are modelled using the orifice equation [56], as shown in Eq. (7). The low-pressure accumulator is essential to avoid pressure drops in the low-pressure line, which could lead to undesirable phenomena such as cavitation. Pressure and volume variations in the accumulator are described via an isentropic and adiabatic process, as described in Eq. (8).

$Q_{v}=C_{d i s} A_{v}(\Delta p) \operatorname{sign}(\Delta p) \sqrt{\frac{2}{\rho_{o i l}}|\Delta p|}$

$p_{a c c}=p_{p r e}\left(\frac{V_{t o t}}{V_{g a s}}\right)^{\gamma}$

where $Q_{v}$ is the flow through the valve, $C_{d i s}$ the valve discharge coefficient, $A_{v}$ the valve opening area, $\rho_{o i l}$ the density of the hydraulic oil, $V_{\text {tot }}$ the total volume of the accumulator, $V_{\text {gas }}$ the gas volume in the accumulator, $p_{\text {pre }}$ the pre-charged pressure of the accumulator, and $\gamma$ the adiabatic index for an ideal gas.

Hydraulic motors convert hydraulic pressure and flow into mechanical torque and rotational speed of the motor shaft coupled to an electric generator. The output flow $\left(Q_{M}\right)$ and torque of the motor $\left(T_{M}\right)$ can be described, respectively, as follows,

$Q_{M}=\alpha D_{\omega} \omega_{M}-Q_{\text {losses }}$

$T_{M}=\alpha D_{\omega} \Delta p_{M}-T_{\text {losses }}$ where $\alpha$ is the motor displacement fraction, $D_{\omega}$ the displacement of the hydraulic motor, $\omega_{M}$ the rotational speed of the shaft, $\Delta p_{M}$ the pressure difference across the hydraulic motor, and $Q_{\text {losses }}$ and $T_{\text {losses }}$ represent leakage and friction losses in the hydraulic motor estimated via the Schlösser loss model [57,58]. Further details about the hydraulic transmission model, such as the identification of the parameters of the Stribeck friction model or Schösser loss model, are provided in [55].

With respect to the electric generator, mathematical models for three different electrical generators can be implemented in the HiFiWEC, as presented in [59]. The generator implemented in the present paper is the squirrel cage induction generator (SCIG), following the equivalent two-phase $(d q)$ representation in [60],

$V_{s d}=R_{s} i_{s d}-\omega \lambda_{s q}+L_{s} \frac{d}{d t} i_{s d}+L_{m} \frac{d}{d t}\left(i_{s d}+i_{r d}\right)$

$V_{s q}=R_{s} i_{s q}+\omega \lambda_{s d}+L_{s} \frac{d}{d t} i_{s q}+L_{m} \frac{d}{d t}\left(i_{s q}+i_{r q}\right)$

$V_{r d}=R_{r} i_{r d}-\left(\omega-\omega_{r}\right) \lambda_{r q}+L_{r} \frac{d}{d t} i_{r d}+L_{m} \frac{d}{d t}\left(i_{s d}+i_{r d}\right)$

$V_{r q}=R_{r} i_{r q}+\left(\omega-\omega_{r}\right) \lambda_{r d}+L_{r} \frac{d}{d t} i_{r q}+L_{m} \frac{d}{d t}\left(i_{s q}+i_{r q}\right)$

where $V$ is the voltage, $i$ the current, $R$ the resistance and $\lambda$ the flux. Subscripts $s$ and $r$ are used for the stator and rotor, respectively, while $d$ and $q$ refer to the direct and quadrature axes, respectively. $\omega$ and $\omega_{r}$ are the angular speed of the reference frame and the rotor, respectively. The model for the SCIG is obtained by setting $V_{r d}=V_{r q}=0$ in Eqs. (13) and (14).

The electromagnetic torque $\left(T_{e}\right)$, rotational speed of the generator shaft and the electric power generated are given, respectively, in Eqs. (15)-(17),

$T_{e}=\frac{3 N_{p}}{4}\left(\lambda_{s d} i_{s q}-\lambda_{q s} i_{d s}\right)$

$\dot{\omega}_{r}=\frac{N_{p}}{2 J}\left(T_{e}-T_{M}-B_{\text {wind }} \omega_{r}\right)$,

$P_{e}=\frac{3}{2}\left(V_{s d} i_{s d}+V_{s q} i_{s q}\right)$

where $N_{p}$ is the number of poles in the generator, $J$ the shaft moment of 
inertia and $B_{\text {wind }}$ the friction/windage damping.

The mathematical PTO model described in this section, including the hydraulic system and the electric generator, has been validated against experimental data generated in different hydraulic test-rigs and electric generators in $[55,32,59]$, respectively.

\subsection{Platform coupling}

The software communication between the OpenFOAM and MATLAB $^{\circledast}$ environments, enabling the coupling of the CNWT and PTO models, is achieved following similar procedures reported in the literature $[38,61]$. The method was first demonstrated in [61], where mooring forces on a floating WEC are calculated in MATLAB $^{\circledR}$ at each time-step of an OpenFOAM CNWT simulation. Similarly, OpenFOAM and $\mathrm{MATLAB}^{\circledR}$ are coupled in [38] for the evaluation of energy maximising control strategies, where an adaptive receding horizon pseudospectral control algorithm is implemented in MATLAB ${ }^{\circledR}$ to calculate the optimal PTO force applied to the WEC at each CNWT time-step. The present paper follows these pioneering couplings of CNWT simulations with mooring models and control algorithms, and presents a first coupling of a full PTO model with a CFD model.

The coupling is driven by the CNWT model, where at each time-step the CNWT simulation pauses, passes information (time, position and velocity values at that time-step) to the PTO model and waits for the PTO model to calculate and return the PTO force at that time-step, before continuing. The CNWT adds the PTO force to the sum of fluid and gravity forces acting on the WEC, to determine the total force from which the acceleration of the WEC and its resulting motion is calculated, using the sixDoFRigidBodyMotion solver in OpenFOAM.

Due to the differences between the time-integration solvers, and the size of the time steps, in the CNWT and the PTO models, interpolation of the variables going through the communication channel is required. The interpolated variables are illustrated with the addition of a hat symbol in Fig. 2. Similar interpolation procedures are also required in the PTO model due to the multi-rate solver [32], where linear interpolation is demonstrated to be sufficiently accurate and computationally efficient. Therefore, linear interpolation is also used in the coupling between the CNWT and the PTO model. A verification test is conducted in Section 4.1 to certify the satisfactory performance of the coupling.

\section{Case study}

A case study is presented to demonstrate the performance of the HiFiWEC. The case study considers a point absorber WEC with a spherical geometry, restricted to heave motion for the sake of simplicity, as illustrated in Fig. 4. The spherical geometry is interesting for the present study, due to its non-uniform cross-sectional area that leads to nonlinear Froude-Krylov (FK) forces [62], requiring a high-fidelity hydrodynamic model to capture this nonlinear effect. A full scale device, with a diameter of $5 \mathrm{~m}$, is chosen to demonstrate the capabilities of the platform, since such a device, and the associated full-scale waves, cannot be physically tested in an experimental wave tank facility due to its size, and testing a full scale device in the ocean during the early design development stages is prohibitively expensive and allows no control over the input wave conditions. Further characteristics of the WEC are given in Table 1.

\subsection{Test cases}

Two test cases are considered:

1. A verification test case, to ensure the coupling between the CNWT and PTO model is implemented correctly and performing as expected.

2. An evaluation test case, to demonstrate the value of the high-fidelity simulation platform offered by the HiFiWEC, for numerical

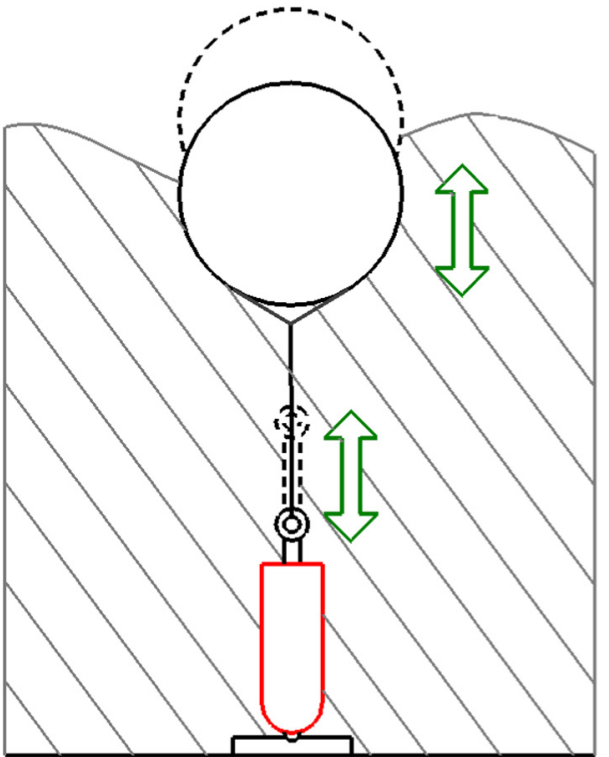

Fig. 4. Schematic of the spherical heaving point absorber considered in the case study.

Table 1

Test case characteristics of the WEC, PTO, input waves and control parameters.

\begin{tabular}{|c|c|c|c|}
\hline & & Verification test & Evaluation test \\
\hline \multirow[t]{3}{*}{ WEC } & WEC diameter & \multicolumn{2}{|c|}{$5 \mathrm{~m}$} \\
\hline & WEC mass & \multicolumn{2}{|c|}{$33.3 \mathrm{~T}$} \\
\hline & WEC natural period & \multicolumn{2}{|c|}{$3.17 \mathrm{~s}$} \\
\hline \multirow[t]{6}{*}{ PTO } & $\begin{array}{l}\text { Hydraulic system time- } \\
\text { step }\end{array}$ & \multicolumn{2}{|c|}{$1 \mathrm{~ms}$} \\
\hline & $\begin{array}{c}\text { Electrical system time- } \\
\text { step }\end{array}$ & \multicolumn{2}{|c|}{$50 \mu \mathrm{s}$} \\
\hline & Cylinder piston area & \multicolumn{2}{|c|}{$140 \mathrm{~cm}^{2}$} \\
\hline & Cylinder length & \multicolumn{2}{|c|}{$2 \mathrm{~m}$} \\
\hline & Motor displacement & \multicolumn{2}{|c|}{$1120 \mathrm{cc}$} \\
\hline & Generator rated power & \multicolumn{2}{|c|}{$74.5 \mathrm{~kW}$} \\
\hline \multirow[t]{3}{*}{ Wave } & Wave type & Monochromatic & Polychromatic \\
\hline & Wave period & $12 \mathrm{~s}$ & $8 \mathrm{~s}$ \\
\hline & Wave height & $0.5 \mathrm{~m}$ & $1.5 \mathrm{~m}$ \\
\hline $\begin{array}{l}\text { Resistive } \\
\text { control }\end{array}$ & ВРTO & $100 \mathrm{kN} \mathrm{s} / \mathrm{m}$ & $170 \mathrm{kN} \mathrm{s} / \mathrm{m}$ [63] \\
\hline Reactive & ВРTO & N/A & $90 \mathrm{kN} \mathrm{s} / \mathrm{m}[63]$ \\
\hline control & $K_{\text {РTO }}$ & $\mathrm{N} / \mathrm{A}$ & $-125 \mathrm{kN} / \mathrm{m}[63]$ \\
\hline
\end{tabular}

assessment of a WEC performance.

\subsubsection{Verification test case}

The verification of the coupling is carried out by comparing results from the HiFiWEC to those obtained from the NLBEMW2W model, which is implemented exclusively in MATLAB and therefore does not require inter-software coupling as in the HiFiWEC. The mathematical model for the PTO system in the NLBEMW2W model is the same highfidelity model as in the HiFiWEC, so any differences in results will arise due to differences in the hydrodynamic models or errors in the coupling of the HiFiWEC. The BEM-based hydrodynamic model is briefly described in Appendix A, and the purely linear version of the model is used in this test case. Therefore, by choosing input wave conditions that ensure the hydrodynamic response of the WEC is sufficiently linear, allows any discrepancy between the HiFiWEC and NLBEMW2W simulations to be attributed to erroneous model coupling in the HiFiWEC.

To ensure linear hydrodyanmic WEC behaviour, it is important to 
minimise the variations in the wetted surface area, resulting in a perfect wave following WEC. This condition can be met with long, waves of small amplitude whose period is far from the WEC natural period ( $3.17 \mathrm{~s})$. However, the longer the wave, the higher the computational cost of the CNWT simulation, since a longer tank is required to ensure that waves are adequately generated and absorbed. Therefore, a monochromatic wave of $12 \mathrm{~s}$ period and $0.5 \mathrm{~m}$ height, illustrated in Fig. 6(a), is selected as a reasonable trade-off between wavelength and computation time, while ensuring linear WEC behaviour in the verification test. Similarly, the PTO force applied to the WEC should not enhance the relative motion between the WEC and the water surface. However, a realistic PTO force, achievable by the PTO system utilised in the case study, needs to be applied in order to properly test the PTO CNWT model coupling. A linear PTO damper with a coefficient of $100 \mathrm{kN}$ is used and found to ensure proper operation of the PTO system and linear hydrodynamic behaviour of the WEC.

To confirm that the selected input wave and PTO control strategy ensure a linear behaviour of the WEC, the degree of nonlinearity of the verification test case is evaluated with the nonlinearity measure suggested in [64]. This measure analyses the degree of nonlinearity using system input $(\eta)$ and outputs $\left(z, \dot{z}\right.$ or $\left.P_{\text {elec }}\right)$ and provides a value between 0 and 1 , where 0 corresponds to perfect linear behaviour. The nonlinearity measure obtained for the verification test is 0.02 , which confirms that the verification test is practically linear.

\subsubsection{Evaluation test case}

The evaluation test case considers a more realistic input wave signal: a polychromatic wave of $8 \mathrm{~s}$ peak period $(\mathrm{Tp})$ and $1.5 \mathrm{~m}$ significant wave height (Hs), that corresponds to the sea-state with the highest occurrence in actual open-ocean sites, such as BIMEP in the Bay of Biscay or Lisbon in the Atlantic Ocean [65]. The polychromatic wave is generated using the idealised JONSWAP spectrum [66]. Fig. 6(b) illustrates the polychromatic wave used in the evaluation test case.

To evaluate the HiFiWEC, it is compared against four other W2W models, listed in Table 2, which combine different approaches to model the hydrodynamic WSI and the PTO system. In addition to the CNWT approach, the hydrodynamic WSI is modelled using both linear and nonlinear BEM-based models (described in Appendix A), where the latter includes nonlinear Froude-Krylov forces and a quadratic viscous damping term (with a drag coefficient, $C_{d}$ ). The PTO system is modelled using both the high-fidelity PTO model presented in Section 2.2 and an ideal PTO model. In the case of the ideal PTO, the mathematical model presented in Section 2.2 is replaced with Eq. (18), only considering the force applied from the PTO system to the absorber and neglecting all the dynamics, losses and constraints of the PTO system. The coupling of the W2W models, which include the CNWT, is the same as that described in Section 2.3 for the HiFiWEC. However, the W2W models that represent hydrodynamic WSIs via BEM-based models do not require any cross-coupling, since the hydrodynamic and PTO models are both implemented in MATLAB.

The WEC performance is assessed using two different control

Table 2

W2W models compared against the HiFiWEC in the evaluation test case.

\begin{tabular}{cc}
\hline Model & Description \\
\hline CNWT + iPTO & A CNWT coupled to an ideal PTO model \\
$L B E M+i P T O$ & Linear BEM-based hydrodynamic model coupled to an ideal \\
PTO & Partially nonlinear BEM model, with $C_{d}=1$ and a high- \\
$N L B E M W 2 W_{C d=1}$ & fidelity PTO \\
$N L B E M W 2 W_{C d=2}$ & Partially nonlinear BEM model, with $C_{d}=2$ and a high- \\
& fidelity PTO \\
\hline
\end{tabular}

strategies: resistive and reactive control. The resistive control strategy passively absorbs the energy from ocean waves by using the PTO as a linear damper, whereas reactive control actively brings the WEC to resonance with the incident ocean waves, maximizing energy absorption, by applying reactive power to the WEC through the PTO. The PTO force is defined by:

$F_{\text {PTO }}(t)=-B_{\text {PTO }} \dot{z}(t)-K_{P T O} z(t)$,

where $B_{\text {РTO }}$ and $K_{\text {РTO }}$ are the PTO damping and stiffness parameters, respectively, with $K_{P T O}=0$ in the case of resistive control. Note that, when an ideal PTO model is employed, the losses and dynamics of the PTO system are not included, therefore the hydrodynamic absorbed power and the generated electrical power are identical, given by:

$P_{e}(t)=-\dot{z}(t) F_{\text {РTO }}(t)$

The optimal $B_{P T O}$ and $K_{P T O}$ are listed in Table 1, and are taken from [63], where control parameters are optimized for the same WEC as considered here, using the $N L B E M W 2 W_{C d=1}$ model. Fig. 5 shows the results of the control parameter optimisation for evaluation test case using the reactive control strategy.

\subsection{Input waves}

The waves are first generated in the CNWT without the WEC in the tank, and the free surface elevation (FSE) at the intended WEC position is recorded (plotted in Fig. 6). The recorded FSE is used as the input wave signal for the BEM-based models, and the CNWT simulations are run again, using exactly the same wave generation settings, with the WEC positioned in the tank. This ensures that results from CNWT- and BEM-based approaches are compared for identical input wave signals and removes the influence of any small errors in wave height or phase stemming from the numerical wave generation in the CNWT.

Analysing the accuracy of the numerical wave generation for monochromatic waves, it is found that the error in wave height at the position of the WEC is below $0.5 \%$, thus the use of CNWT wave data as input for BEM-based models may not necessarily be required. For polychromatic sea states, it is well known that short time traces do not accurately represent the statistical properties of the desired spectrum $[67,68]$. However, for the purpose of this test case, a more realistic input wave signal than a monochromatic wave is desired, and perfect representation of the spectrum is not required, since the CNWT- and BEM-based models are not compared using statistical properties, but rather directly using their time domain outputs.

\subsection{CNWT setup}

Details of the CNWT geometry, mesh and time-steps for the two test cases are now presented.

\subsubsection{Tank geometry}

For both test cases, the WEC is positioned in the centre of a simulation zone of 10 WEC radii $(25 \mathrm{~m})$ length and $100 \mathrm{~m}$ width. However, the water depth and the creation and absorption zone lengths are different for each test case, as listed in Table 3, due to the different characteristic wavelengths considered.

The length of the wave creation and absorption zones are parameterised by the wavelength, being 1.5 and 3 wavelengths long, respectively. These values are selected based on the parameter study in [67], which considers the same sea state as in the evaluation test case. For the absorption zone, increasing the length decreases wave reflection and, at a length of three times the peak wavelength, the reflection coefficient drops below $1 \%$. For the wave creation zone, the parameter study considers a regular wave with the same period as the peak period $(8 \mathrm{~s})$ and the same height as the significant wave height $(1.5 \mathrm{~m})$. A wave creation zone of 1-1.5 wavelengths is found to generate the most accurate waves. Therefore, the longer zone length of 1.5 wavelengths is 


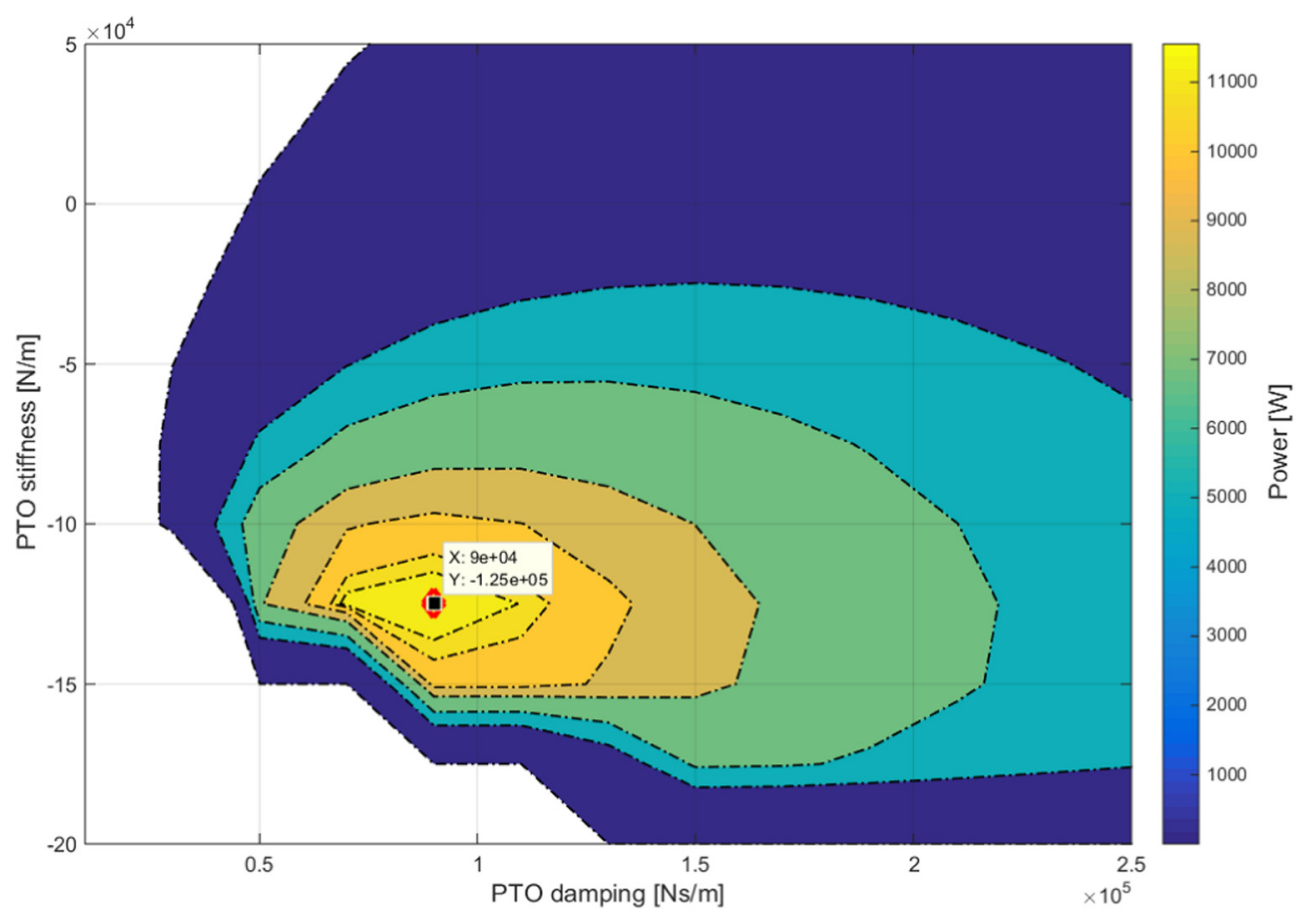

Fig. 5. Reactive control parameter optimisation for the evaluation test case using the $N L B E M W 2 W_{C d=1}$ approach [63].

chosen, to allow for good absorption of radiated waves from the WEC travelling back towards the wave creation zone and to accurately generate waves in the polychromatic spectrum with wavelengths longer than the peak wavelength.

The verification test case considers a $12 \mathrm{~s}$ regular wave, which has a wavelength of $225 \mathrm{~m}$, whereas the evaluation case considers a wave spectrum with a peak period of $8 \mathrm{~s}$, which has a peak wavelength of about $100 \mathrm{~m}$. The water depth of $70 \mathrm{~m}$, for the evaluation test, is chosen to be representative of the depth at the BIMEP test site in the Gulf of Biscay. However, in the verification test, for the $12 \mathrm{~s}$ regular wave, the $70 \mathrm{~m}$ water depth does not correspond to deep water and the resulting wave would not be regular; therefore, the water depth is increased to approximately $70 \%$ of the wavelength to ensure deep water conditions.

The computational domain includes a $25 \mathrm{~m}$ height above the equilibrium water level to the atmospheric boundary. To reduce the computational requirement, a symmetry plane is utilised through the centre
Table 3

Numerical wave tank characteristics.

\begin{tabular}{cccc}
\hline Test case & Creation zone & Absorption zone & Water zone \\
\hline Verification & $337.5 \mathrm{~m}$ & $675 \mathrm{~m}$ & $160 \mathrm{~m}$ \\
Evaluation & $150 \mathrm{~m}$ & $300 \mathrm{~m}$ & $70 \mathrm{~m}$ \\
\hline
\end{tabular}

of the tank, so that only half of the tank and WEC width is simulated. The influence of the symmetry plane on the body motion is investigated in [54] and found to be negligible.

\subsubsection{Mesh}

The mesh is depicted in Fig. 7. Vertically, the mesh consists of three regions, corresponding to the air, water and interface regions. The interface region has a height of $10 \mathrm{~m}$, centred at the still water level, and

(a) Verification test $-\mathrm{Tw}=12 \mathrm{~s} \& \mathrm{Hw}=0.5 \mathrm{~m}$

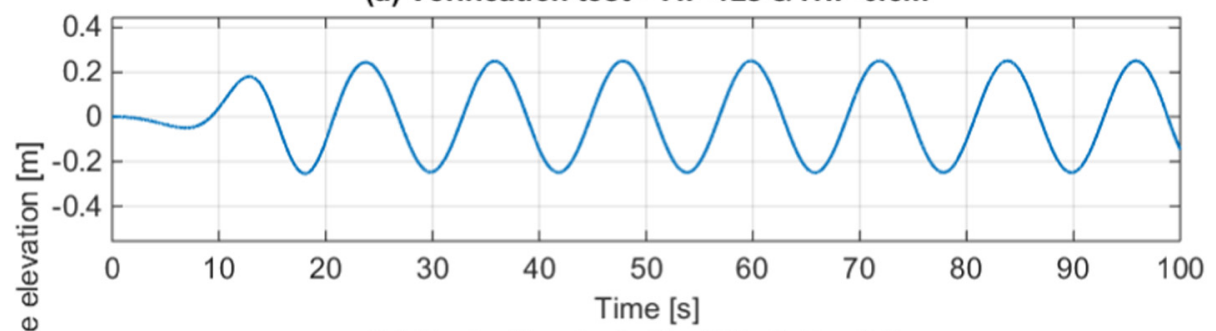

(b) Evaluation test $-\mathrm{Tp}=8.5 \mathrm{~s} \& \mathrm{Hs}=1.5 \mathrm{~m}$

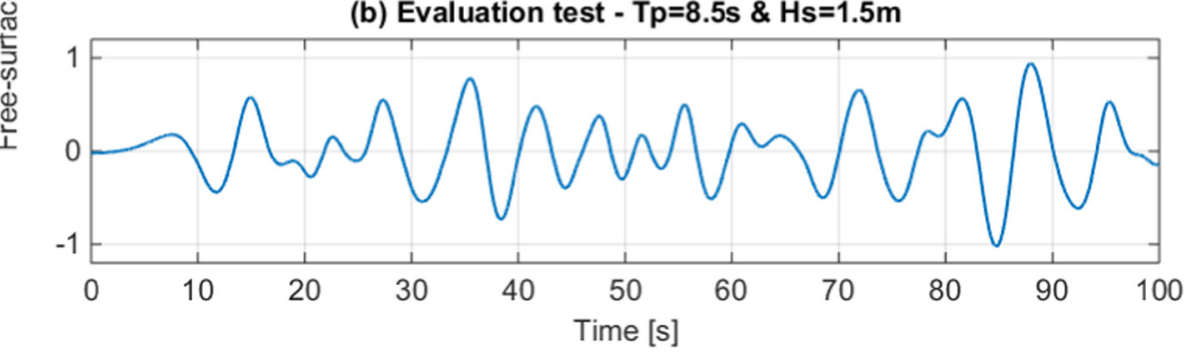

Fig. 6. Input waves for the two test cases: monochromatic waves for the verification test (a) and polychromatic waves for the evaluation test (b). 


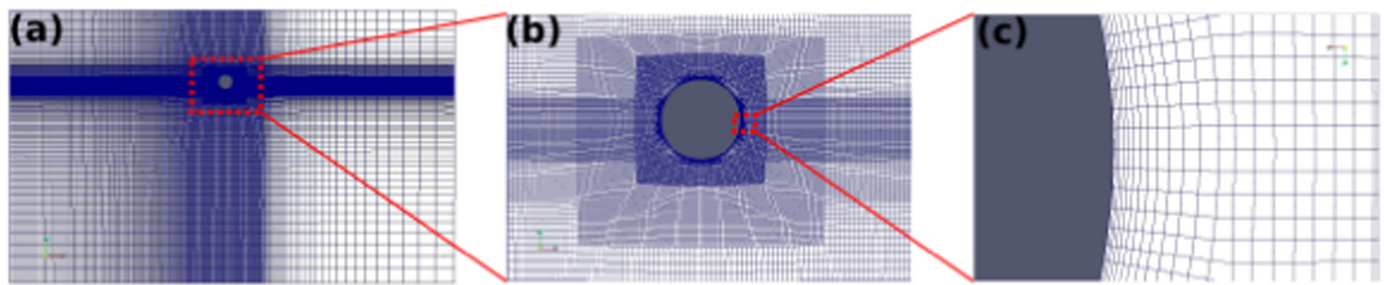

Fig. 7. (a) The CNWT mesh. (b) Zoom in of mesh around the WEC. (c) Further zoom in of mesh refinement around body to ensure adequate yPlus values.

meshed vertically with uniform cells of height $\Delta C$. These cells are then split in half at a distance of $2.5 \mathrm{~m}$ above and $4.5 \mathrm{~m}$ below the still water level (SWL), and then split into half again $2 \mathrm{~m}$ either side of the SWL, so that the cells in the region where the free surface will be generated have a height of $\Delta C / 4$.

Adjacent to the interface region, the mesh is stretched vertically towards the boundaries of the tank, using the grading ratio $G R$, defined as the ratio between two subsequent cell sizes in the direction of stretching. In the water region, the mesh is stretched towards the tank floor with $g r=1.075$. In the air region, the mesh is stretched to the atmosphere boundary with $g r=1.2$.

Horizontally, across the width of the tank, the mesh has a width of $\Delta C$ and then is stretched towards the side wall with $g r=1.1$.

Along the length of the tank, the mesh has three regions, corresponding to the creation, simulation and absorption zones. In the simulation zone the mesh has a constant length of $\Delta C$. In the absorption zone, the mesh is stretched towards the downwave tank wall with $g r=1.1$. In the creation zone, the mesh is stretched towards the upwave tank wall, with $g r=1.075$ in the verification test case, and $g r=1.01$ in the evaluation test case. The reason for the difference in $G R$ between the two cases is due to the different wave regimes used in these tests. It is important to have a specific number of cells per wave length, which can easily be determined for a regular wave. However, since a wave spectrum contains a range of frequencies, including high frequency waves with short wavelengths, the cell length needs to be smaller for these shorter waves. Therefore, a much smaller $G R$ is used for the wave spectrum case, to capture the high frequency waves in the spectrum.

The GR values for the different regions are determined using twodimensional (2D) trial runs, whereby the tank is only one cell thick, to reduce the overall cell count and allow many fast simulations. The $G R$ was incremently increased until the solution began to diverge, to identify values that provide accurate solutions while ensuring the least amount of cells are used, which is vital for the subsequent three-dimensional simulations.

The mesh in the region around the WEC was also refined, as shown in Fig. 7(b). The cell lengths were split in half in all directions within a box extending a horizontal distance of three WEC radii from the centre of the WEC, and a vertical distance of three WEC radii downwards and two WEC radii upwards from the centre of the WEC. A second refinement box then split the cell lengths in half again, extending a distance of 1.5 WEC radii in all directions, so that the mesh in the box around the WEC has cubic cells of length $\Delta C / 4$. During this refinement procedure, the vertical cell lengths in the already refined free surface regions remained unchanged.

A mesh convergence study was undertaken, to determine an appropriate value for $\Delta C$, considering the wave generation and propagation for the wave spectrum, in the evaluation test case. Three mesh resolutions were investigated, where $\Delta C$ was doubled between each mesh, resulting in a coarse, medium and fine mesh, with $\Delta C$ of $0.625 \mathrm{~m}, 0.3125 \mathrm{~m}$ and $0.15625 \mathrm{~m}$, respectively (these values were chosen as they are factors of $5 \mathrm{~m}$ allowing clean division of the simulation and interface regions). The resulting FSE measured at the centre of the simulation zone is plotted in Fig. 8, with very little difference observed between the three meshes. However, as shown in the zoom box, the results from the coarse mesh diverges slightly, at some wave peaks and troughs, from the other two meshes which are always in good agreement with each other. Therefore, the medium mesh, with $\Delta C=0.3125 \mathrm{~m}$ is used for the case study. This relates to 19.2 cells per waveheight, which is consistent with predominant values reported in [13].

The final mesh parameter to be determined is the length of the first cell layer adjacent to the WEC, which can be adjusted using surface layers growing from the WEC to the background mesh, as depicted in Fig. 7(c). The length of the first cell layer, in conjunction with the fluid velocity in the cell, determines the $y^{+}$value, which is important for the performance of the wall functions used within the turbulence model. The recommended y-Plus values are $30<y^{+}<500$, for fully developed turbulent flows in single phase fluids, which is challenging to apply to the case of WECs, since it involves oscillating flows with a free surface. The pragmatic approach generally taken when applying turbulence models to WECs [13], is to perform a sensitivity analysis of the results to these parameters. In the present mesh study, refinement around the WEC with an expansion ratio of 1.2 from the WEC body to the background mesh is used. The results for two different meshes are shown in Fig. 9, where Mesh 1 uses 12 refinement layers, resulting in a first cell thickness of $0.010 \mathrm{~m}$, and Mesh 2 uses 9 refinement layers, resulting in a first cell thickness of $0.018 \mathrm{~cm}$. The $y^{+}$values are calculated every $2 \mathrm{~s}$ (since this requires cell values to be written to memory at each time step, increasing memory requirements and computation time), where the minimum and maximum values from all the cells surrounding the WEC are recorded. Mesh 1 has a range of $y^{+}$values at each time-step, with minima of 16-64 and maxima of 211-478. Mesh 2 has values with minima ranging from 44 to 114 and maxima from 410 to 835 . Therefore, in some instances, Mesh 1 has $y^{+}$values that are too low, whereas Mesh 2 has some that are too high; however, comparing the outputs from the two simulations shows that there is very little sensitivity, with differences in WEC motion of less than $0.2 \%$ at the peaks/troughs. Therefore, Mesh 1 with a first cell thickness of $0.01 \mathrm{~m}$ is used for the case study.

Overall, 1.3 million cells are used for the verification test setup and 1.7 million cells for evaluation test setup. Although the tank geometry is larger for the verification test, the greater mesh stretching used in the creation zone gives an overall lower number of cells, compared to the evaluation test case.

\subsubsection{Time steps}

A constant time step of $0.01 \mathrm{~s}$ is used in all simulations. While OpenFOAM offers the option of an adaptive time step, to ensure adherence to a maximum specified Courant number, the constant time step is chosen due to an observed time step dependence of the generated waves from waves2Foam. Since smaller mesh cells lead to smaller time steps under the adaptive time step scheme, slightly different input waves are generated by waves2Foam for cases with different meshes. Therefore, a mesh convergence study is impossible using an adaptive time step with the waves2Foam toolbox. Additionally, when the WEC is introduced into the tank, small mesh cells are used to capture the boundary layer around the WEC; therefore, using the adaptive time step approach, the time step used in the WEC simulation will be different from the waves-only case, meaning that the generated waves will be slightly different in the two cases. 


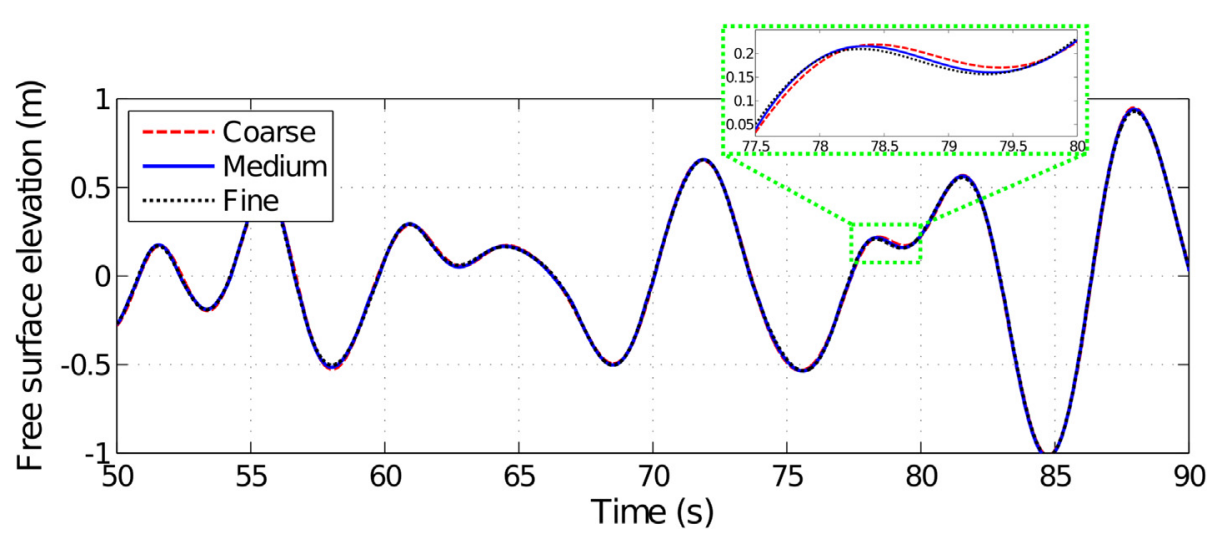

Fig. 8. Free surface elevation for increasing mesh refinement.

Consequently, in addition to a spatial mesh convergence study, a time step convergence study is also performed. For wave propagation, due to the time step dependence of the waves2Foam wavemaker, the results from the time step convergence study in [67] are observed, which used the static boundary method for wave generation. Windt et al. [67] considers a regular wave with $8 \mathrm{~s}$ period and $1.5 \mathrm{~m}$ wave height, on a mesh with the same resolution as considered here (20 cells per waveheight), and finds a converged time step value of $0.02 \mathrm{~s}$.

For the present study, a further time step convergence study is required for the body motion/fluid structure interaction solution. Since the body (WEC) cannot be driven by the waves, due to the time step dependence of the wavemaker, the convergence study opts to drive the body with the PTO force. To ensure representative dynamics as are expected in the test cases, the PTO force signal generated by the NLBEMW2W model, in the reactive control case study, is used. Time step values of $0.04 \mathrm{~s}, 0.02 \mathrm{~s}, 0.01 \mathrm{~s}$ and $0.005 \mathrm{~s}$ are investigated, and the results plotted in Fig. 10, from which a time step of $0.01 \mathrm{~s}$ is selected.

Using the time step of $0.01 \mathrm{~s}$, the maximum Courant number is monitored during the evaluation test case simulations and observed to range between 0.15 and 0.6 .

\subsection{Power take-off system design}

The design of the PTO system is characterised by the pressure and flow requirements of the hydraulic system to absorb energy from ocean waves. The main parameters of the PTO, presented in Table 1, are the cylinder piston area and motor displacement. These two parameters are defined following three main constraints of a WEC: maximum pressuredifference in the cylinder chambers, and maximum WEC displacement and velocity.

Pressure should be as high as possible to minimize losses. However, the efficiency of a hydraulic motor, according to the efficiency map shown in [55], reduces with pressures above 300 bar, so the maximum pressure difference allowed in the system is set at 300 bar. Hence, the hydraulic cylinder piston area $\left(A_{p}\right)$ is designed so that the maximum force is provided when the pressure difference between the two cylinder chambers is 300 bar. The maximum allowable force for the WEC defined in Section 3 is $420 \mathrm{kN}$, based on the requirements defined in [69] for a similar device, which results in a piston area of $140 \mathrm{~cm}^{2}$.

WEC displacement and velocity constraints substantially influence the power absorption of the WEC [70-72] and, thus, the WEC and PTO system should be carefully designed. However, the optimization of the PTO is beyond the scope of the present study. Therefore, two typical values are considered for WEC displacement and velocity constraints, $2 \mathrm{~m}$ and $2 \mathrm{~m} / \mathrm{s}$, respectively. The velocity constraint determines the maximum possible hydraulic fluid flow that can be used to determine the required motor displacement. Assuming the PTO operates at a fixedspeed of $1500 \mathrm{rpm}$, a hydraulic motor of $1120 \mathrm{cc}$ displacement is required.

Selection of the electric generator is particularly complex due to the highly irregular output power signal of WECs. The rated power of the generator needs to be large enough to follow the highest absorbed power peaks, while keeping reasonable efficiency in low energy seastates. In this case, a generator of $74.5 \mathrm{~kW}$ has been selected, using the parameters of the electric generator provided in [73].

\section{Results}

Results for the verification test case are presented first in Section 4.1, confirming the correct implementation and performance of the model coupling within the HiFiWEC. Following that, the results of the evaluation test case are presented in Section 4.2, demonstrating the merit of the HiFiWEC.

\subsection{Verification test case}

Results for the verification test case are shown in Fig. 11. The WEC heave displacement and the PTO force are plotted in Fig. 11(a) and (b),

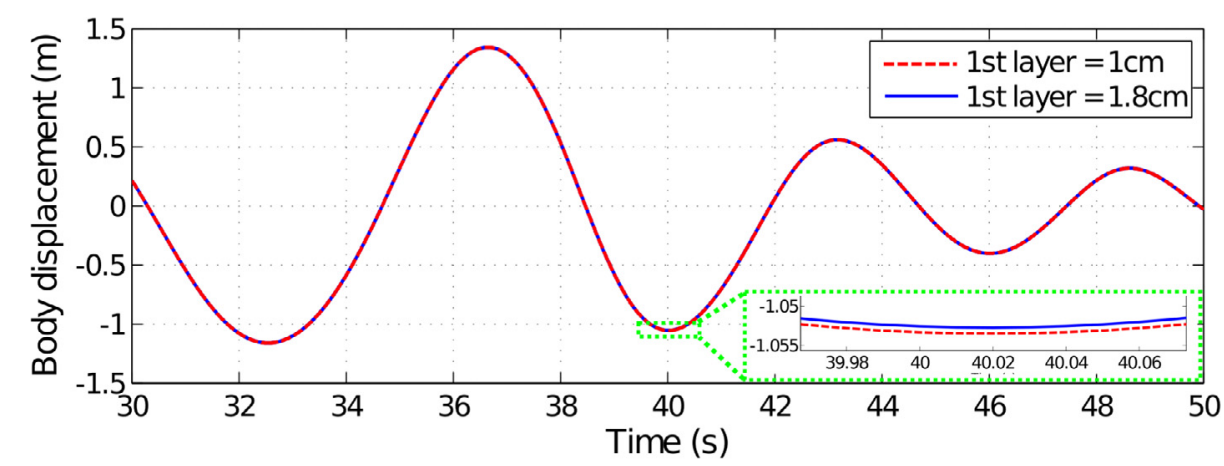

Fig. 9. WEC heave displacement for different mesh resolution around the body. 


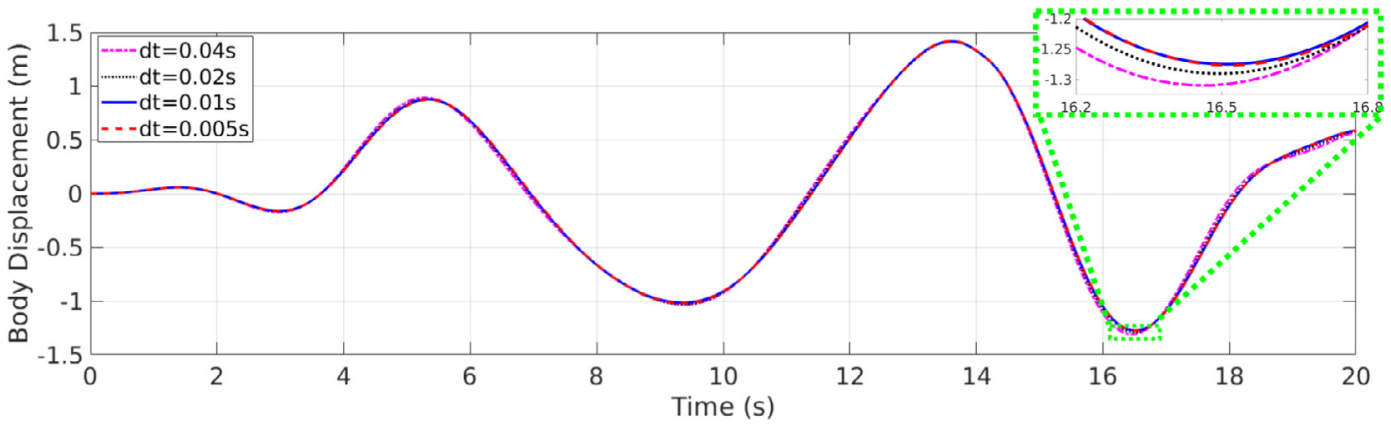

Fig. 10. WEC heave displacement for varying time step lengths.

respectively, showing that the HiFiWEC and the LBEMW2W model agree extremely well for simulation times $t>30 \mathrm{~s}$.

The difference within the first $30 \mathrm{~s}$ arises due to the way in which the BEM-based model interprets the input wave signal. The hydrodynamic coefficients for the BEM-based model are calculated using a frequency domain approach, where the excitation on a body for each frequency assumes interaction with a fully developed wave field of that single frequency. Therefore, the wave field exists both upwave and downwave from the body. However, for the CNWT, when the wave initially starts to interact with the body due to the upwave pressure field, the fluid pressure on the downwave side of the WEC is still at rest and, thus, the response of the WEC in the CNWT is inferior to that of the linear BEM model.

To complete the verification of the HiFiWEC, generated power signals obtained from the HiFiWEC and the LBEMW2W model are compared in Fig. 11(c). A notable characteristic of the generated power signal, for both models, is the high frequency oscillation during the first $5 \mathrm{~s}$. These oscillations correspond to the start-up of the induction generator, where the generator is accelerating until reaching the synchronous rotational speed. Once the generator is operating at synchronous speed, steady-state is reached around $30 \mathrm{~s}$ in the LBEMW2W model, while it takes about $10 \mathrm{~s}$ longer in the HiFiWEC. However, results again show a perfect match in steady-state. Thus, the correct performance of the coupling in the HiFiWEC is verified.

\subsection{Evaluation test case}

Using the input wave series shown in Fig. 6(b), the WEC hydrodynamic behaviour and power generation simulated by the HiFiWEC is compared against the four other W2W models described in Section 3.1.2. To remove any irrelevant initialisation effects, results are analysed for $t \geqslant 40 \mathrm{~s}$, based on the observations from the verification test case.

The results from the resistive control simulations are shown in Fig. 12. The hydrodynamic behaviour of the WEC, illustrated by the body motion in Fig. 12(a), is similar for all five simulations. However, a

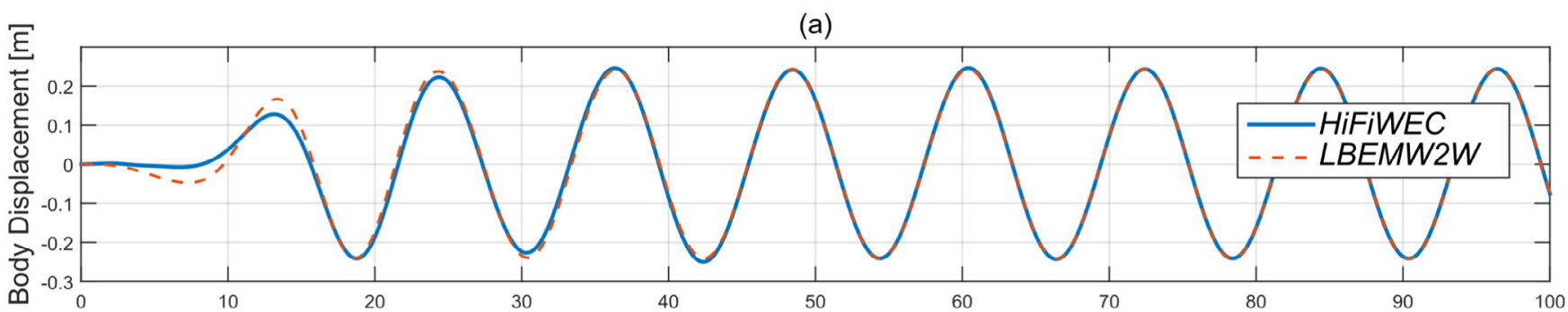

(b)

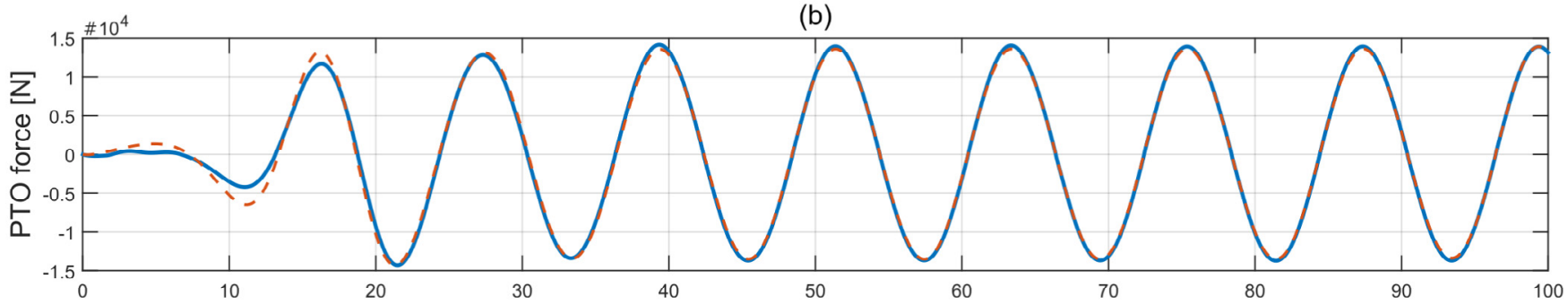

(c)

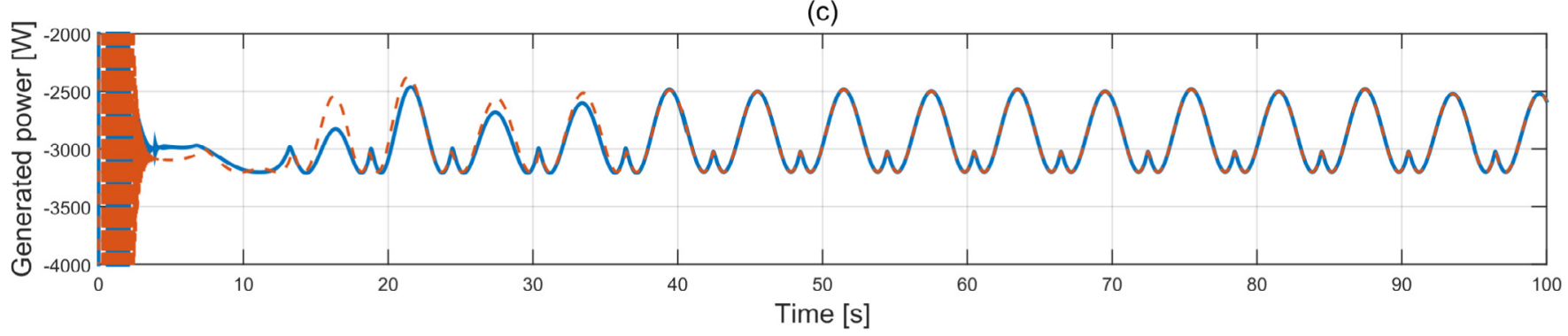

Fig. 11. Verification test case results from the HiFiWEC and the LBEMW2W models for (a) position, (b) PTO force and (c) generated power. 


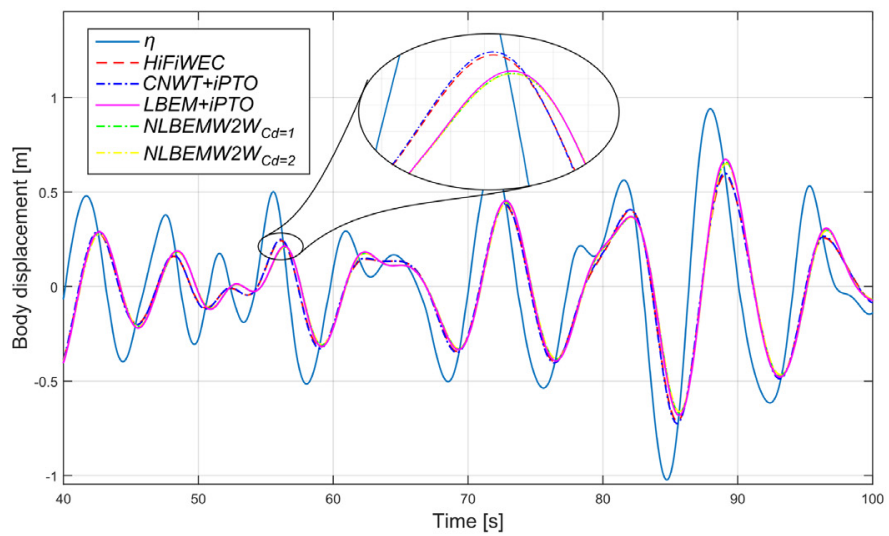

(a)

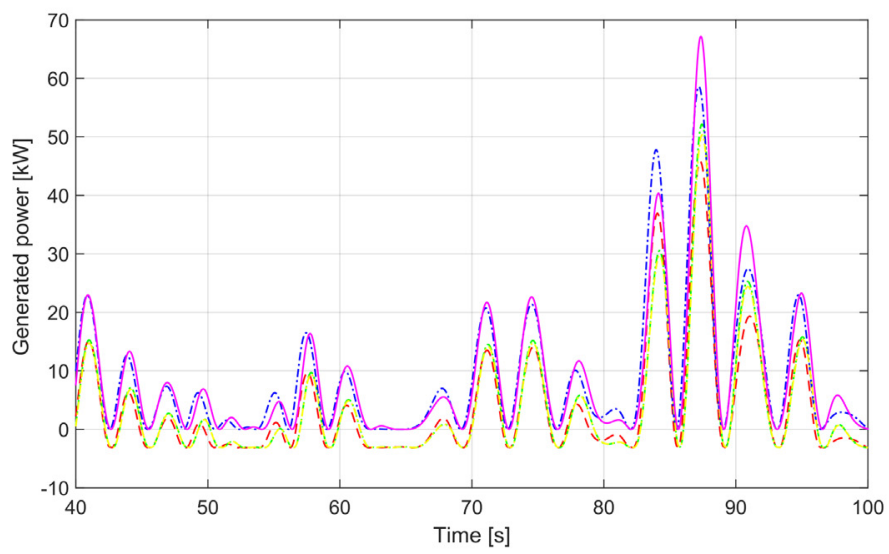

(c)

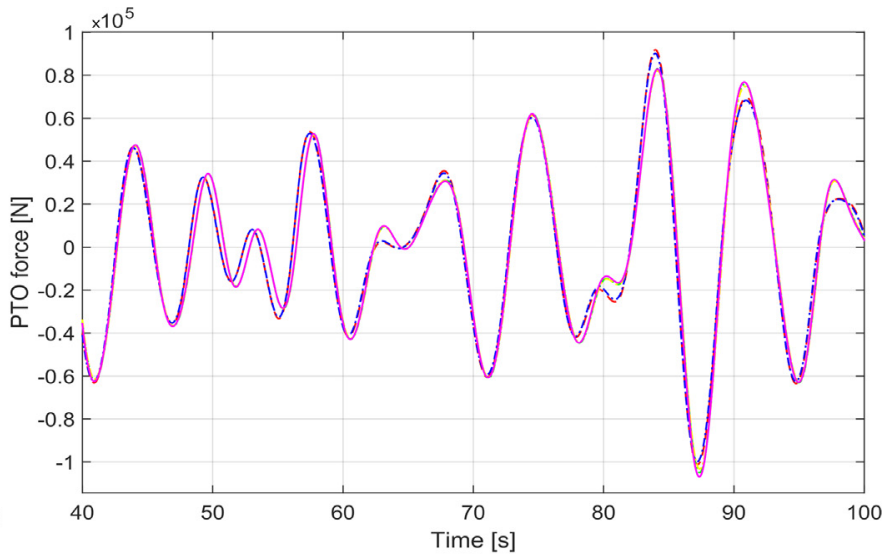

(b)

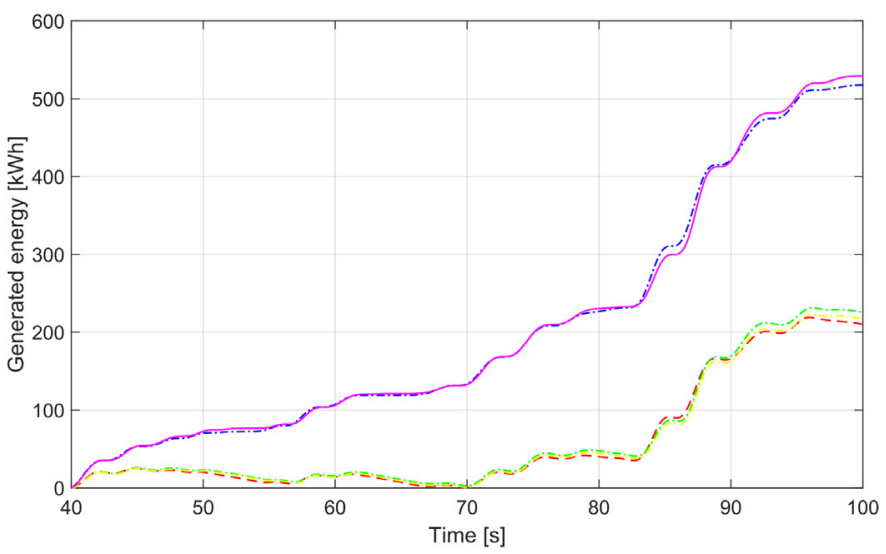

(d)

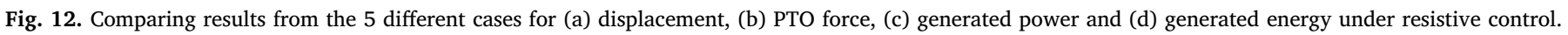

small difference can be seen between the CNWT- and BEM-based approaches, highlighted in the zoomed snapshot in Fig. 12(a). With respect to the PTO force, Fig. 12(b) shows that, under resistive control, the force applied by the PTO system on the absorber is almost identical in all the W2W models. Small discrepancies can be observed between CNWT- and BEM-based approaches, likewise in Fig. 12(a), suggesting that discrepancies in the PTO force are mainly caused by differences in the hydrodynamic model. However, although the impact of the highfidelity PTO model is negligible, with regard to PTO force, it significantly affects the generated power and energy estimates, as illustrated in Fig. 12(c) and (d). For example, generated power signals corresponding to the W2W models with the ideal PTO are always positive, while the power signals corresponding to the W2W models with the high-fidelity PTO reach negative values. Negative values in the W2W model with the high-fidelity PTO model, also seen in [63], appear due to the need to draw energy from the electricity grid to keep the electric generator rotating at synchronous speed $(1500 \mathrm{rpm})$ when the absorbed energy is zero or close to zero. On the other hand, differences in the power peaks between the W2W models with the ideal and the high-fidelity PTO models correspond to energy losses considered in the high-fidelity PTO model, which result in significantly lower generated power peaks. The cumulative generated energy plotted in Fig. 12(d) illustrates that the W2W models with the ideal PTO model significantly overestimate the generated power (by more than 100\%), compared to the results from the W2W models with the high-fidelity PTO model.

Table 4 compares the time-average absorbed and generated power values from the W2W models against the values from the HiFiWEC. Absorbed power refers to the mechanical power directly absorbed from ocean waves, while generated power refers to the final electric power output. Absorbed power mainly depends on the hydrodynamic
Table 4

Time-averaged absorbed and generated power values obtained from the HiFiWEC (in kW) and the percentage difference $(\epsilon)$ of the W2W models.

\begin{tabular}{|c|c|c|c|c|}
\hline \multirow[t]{2}{*}{ Model } & \multicolumn{2}{|c|}{ Resistive control } & \multicolumn{2}{|c|}{ Reactive control } \\
\hline & $P_{a b s}^{a v}$ & $P_{g e n}^{a v}$ & $P_{a b s}^{a v}$ & $P_{\text {gen }}^{a v}$ \\
\hline HiFiWEC $[\mathrm{kW}]$ & 8.60 & 3.50 & 23.76 & 13.99 \\
\hline CNWT + iPTO [E (\%)] & 0.32 & 146.4 & 3.03 & 78.36 \\
\hline$L B E M+i P T O[\in(\%)]$ & 2.50 & 151.8 & 16.32 & 97.52 \\
\hline$N_{L B E M W} 2 W_{C d=1}[\in(\%)]$ & 0.51 & 7.21 & -7.60 & -2.87 \\
\hline$N L B E M W 2 W_{C d=2}[\epsilon(\%)]$ & -1.81 & 3.19 & -14.25 & -11.58 \\
\hline
\end{tabular}

behaviour of the WEC. Table 4 shows that the relative deviation between the HiFiWEC and the other W2W models $(\epsilon)$ in absorbed power, under resistive control, is low, similar to the trend shown in Fig. 12(a). Regarding the generated power, the NLBEMW $W$ models produce results relatively close to the HiFiWEC, with Table 4 reporting relative deviations in the generated energy of $7.21 \%$ and $3.19 \%$ for $N L B E M W 2 W_{C d=1}$ and $N L B E M W 2 W_{C d=2}$, respectively.

Interestingly, although $N L B E M W 2 W_{C d=2}$ yields closer overall average generated power to the HiFiWEC, NLBEMW2 $W_{C d=1}$ yields closer overall absorbed power, highlighting the effect of PTO dynamics between the absorbed and generated energy.

The results from the reactive control simulations are shown in Fig. 13. The hydrodynamic nonlinearities are seen to be enhanced under reactive control, where differences between the body motion simulated by the different W2W models, shown in Fig. 13(a), are more evident compared to the resistive control case. This is also evidenced in Table 4, where $\epsilon$ in absorbed power increases by about an order of 


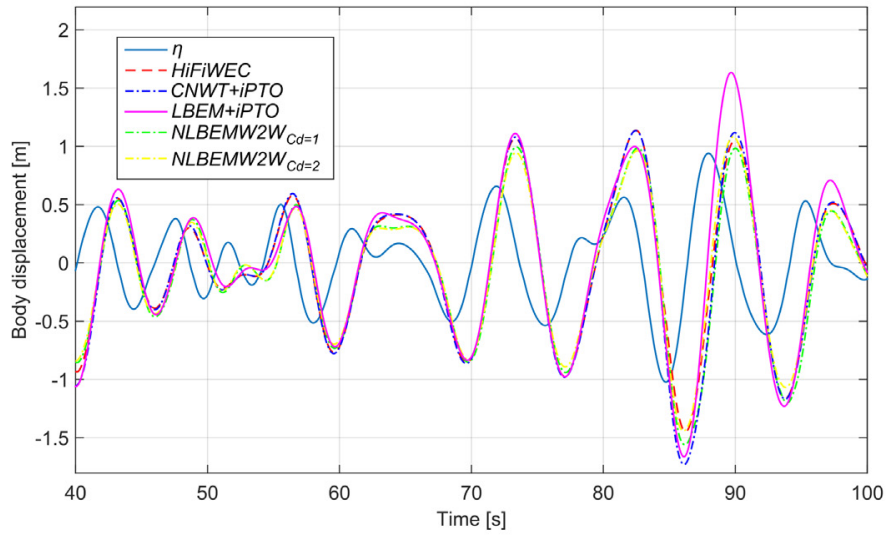

(a)

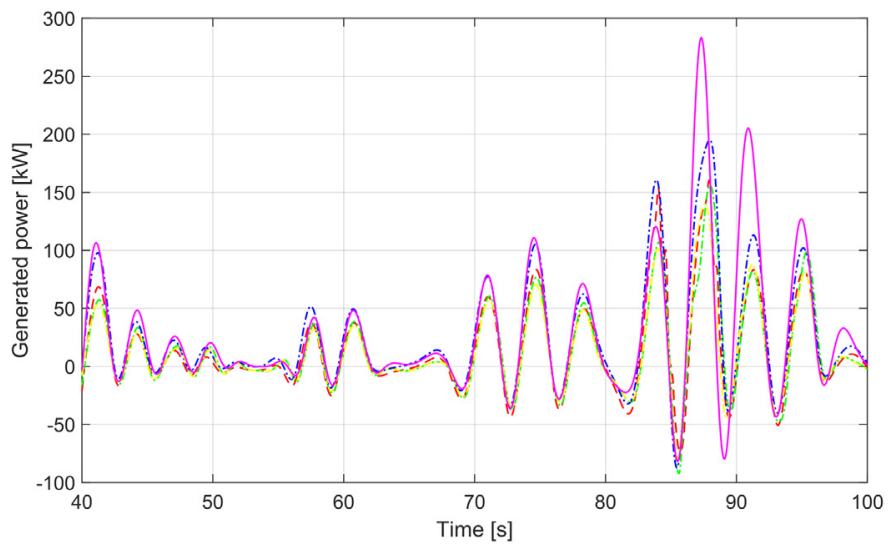

(c)

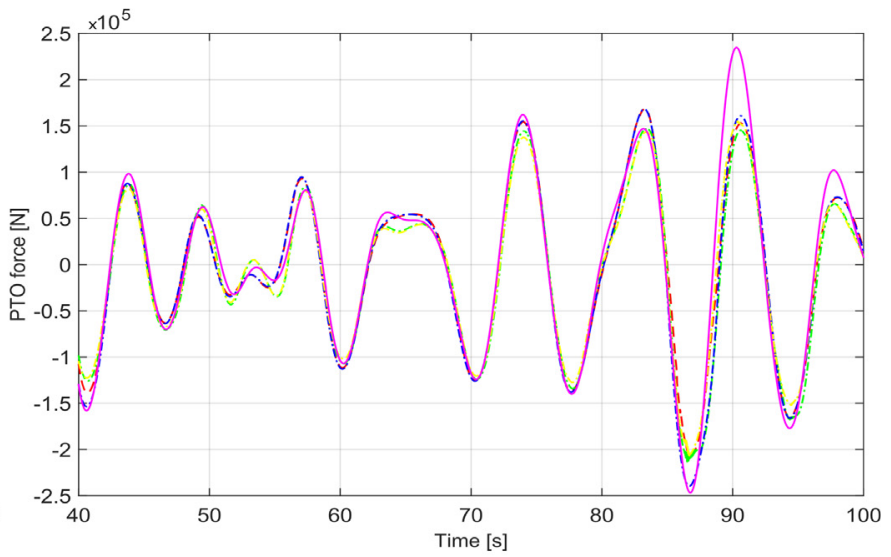

(b)

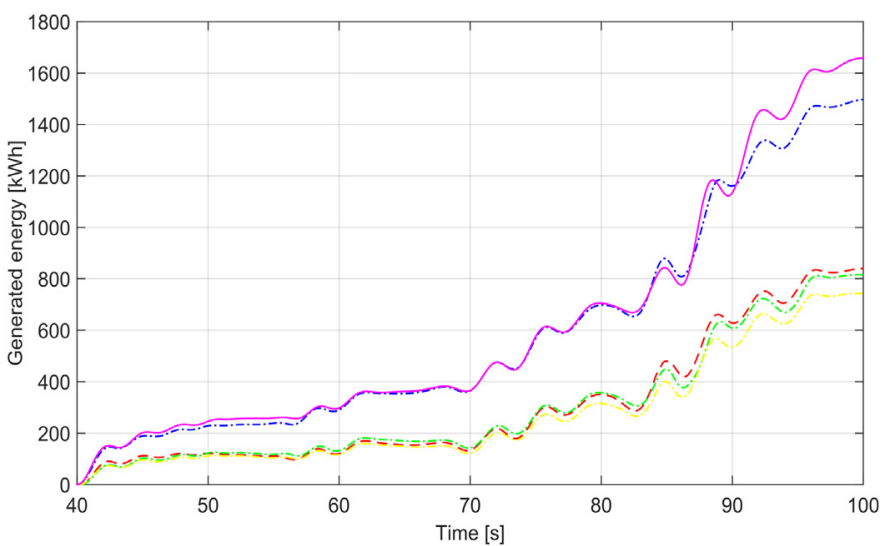

(d)

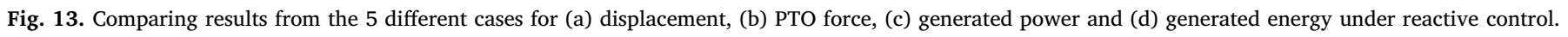

magnitude compared to the relative deviation values for resistive control. Consequently, differences between CNWT- and BEM-based approaches are also more evident in the PTO force profile, compared to the resistive control case, as illustrated in Fig. 13(b).

Overestimation of energy generation under reactive control, illustrated in Fig. 13(d), by the ideal PTO models, compared to the highfidelity PTO models, is not as large as under resistive control. The efficiency of the PTO system is higher under reactive control, where the hydraulic system operates closer to its optimal operation point, due to increased fluid pressure in the hydraulic cylinder and motor. As a consequence, the difference between the high-fidelity and ideal PTO models is smaller compared to the resistive control case. Similarly, generated power signals in Fig. 13(c) also illustrate smaller differences among the different W2W models.

An interesting finding is that, unlike under resistive control, the relative deviation in generated energy under reactive control is lower for $N L B E M W 2 W_{C d=1}$ than for $N L B E M W 2 W_{C d=2}$, which illustrates the inconsistency of the viscous model. In addition, the relative deviation in absorbed energy under reactive control is higher than the deviation in generated energy for $N L B E M W 2 W_{C d=1}$ and $N L B E M W 2 W_{C d=2}$, as opposed to the resistive control case, where the relative deviation in generated energy is always higher. This again shows the inconsistency of the viscous model, which is shown to be particularly inconsistent when the motion of the device is exaggerated via an energy maximising control strategy.

Regarding computational requirements, the runtimes of the HiFiWEC are of the same order of magnitude as the CNWT + iPTO, because the CNWT is the computationally heaviest part in the HiFiWEC. Compared to the BEM-based approaches, both CNWT-based approaches are $\mathscr{O}(1000)$ slower than any $N L B E M W 2 W$ approach and $\mathscr{O}(10,000)$ slower than the $L B E M+i P T O$ model.

\section{Discussion}

The results from the case study highlight the relative defficiencies of the different lower fidelity W2W modelling approaches, compared to the HiFiWEC. The LBEM + iPTO model is the most widely used approach in the literature for evaluating the power production of WECs, due to its simplicity and appealing computational requirements. However, comparison of the generated power outputs from the $L B E M+i P T O$ model, against the results attainable from the HiFiWEC, demonstrate the large relative deviations which can arise from excessively simplifying the hydrodynamic WSI and the PTO system modelling.

Comparing the CNWT + iPTO model with the HiFiWEC, isolates the importance of including a high-fidelity PTO model. The results show that neglecting the dynamics, constraints and losses of the PTO system, can lead to large overestimation in the generated power output, with relative deviations of $146 \%$ and $78 \%$ for the resistive and reactive control cases, respectively. Conversely, comparing the NLBEMW2W models with the HiFiWEC isolates the importance of using the CFD approach to model the hydrodynamics. The results show that using the partially-nonlinear BEM-based approach, which includes nonlinear FK forces and viscous effects, can reduce the relative deviations in absorbed power to $14 \%$, compared to the HiFiWEC. Therefore, for the particular WEC, PTO system and operating cases considered, including the high-fidelity PTO model is seen to have a much larger influence on improving the accuracy of the simulated W2W system compared to including the high-fidelity CFD model.

However, the HiFiWEC not only incorporates the individual benefits 
of high-fidelity WSI and PTO models, but also offers the unique possibility to evaluate the holistic performance of a complete WEC system in high-fidelity. The significance of this is highlighted in Table 4, showing that the relative deviation in NLBEMW2W models alternates from absorbed to generated power, even though the PTO model is identical in the HiFiWEC and the NLBEMW2W models. This is important since it implies that analysing the hydrodynamic WSI and the PTO system independently, even with high-fidelity approaches, does not necessarily provide accurate results.

High-fidelity results are also essential to accurately evaluate the performance of the different control strategies. Apart from the differences among the different modelling approaches, the importance of actively controlling the WEC is clearly illustrated in Table 4, where the average generated power in the reactive control case is shown to be significantly higher (about $40 \%$ higher) than the average absorbed power in the resistive control case. This suggests that actively controlling the WEC will always result in a higher generated power, regardless of the efficiency of the PTO system. In addition, one can observe, in Table 4, that the percentage error for the absorption stage is very low for all the different mathematical models under resistive control, while increasing considerably under reactive control. Therefore, it can be concluded that an accurate representation of hydrodynamic WSIs, including all the nonlinear effects, is particularly important when the device is actively controlled to maximise the energy generation, as suggested in previous studies [34,62].

The price for such a high-fidelity platform is a high computational cost. Comparing the HiFiWEC to the NLBEMW2W models reveals a 1000 -fold increase in run time. However, the results in the case study show a relative deviation in generated output power of $3-12 \%$, which varies depending on the drag co-efficient and on the operating conditions in the test (resistive or reactive control). A similar issue is also identified in [35], where the difficulty in consistently and accurately including viscous effects in BEM-based models is discussed. In this respect, the HiFiWEC can be useful in identifying the best drag coefficients and other parameters to be included in such computationally efficient models, and to evaluate their performance and allow the accuracy range to be ascertained. However, some nonlinear effects can only be captured using fully-nonlinear approaches, so that, under certain circumstances, the performance of the best BEM-based approach may still be inadequate results. Indeed, the heaving sphere analysed in this paper is intentionally simple for demonstrative purposes whereas, in general, many WECs are significantly more complex, with several degrees-of-freedom, complicated geometries and may include mooring lines (in which case the HiFiWEC must be extended to include coupling to high-fidelity mooring models [74]). Finally, for some applications, such as validation/verification, model parameter identification, and control strategy evaluation, computational time is not a crucial requirement, while high-fidelity is vital.
Hence, the HiFiWEC is a vital numerical tool for the design of successful WECs in the early stage of development, where open sea experimental tests are prohibitively expensive. As a complement to physical small-scale wave tank experiments, the HiFiWEC allows for a highfidelity evaluation of the performance of the full-scale WEC, including the dynamics, losses and constraints of the PTO system.

\section{Conclusion}

The paper presents a high-fidelity wave-to-wire platform (HiFiWEC), coupling a CFD-based numerical wave tank with a high-fidelity power take-off model, to accurately simulate the behaviour of wave energy converters from ocean waves to the electrical grid. The HiFiWEC is a valuable tool to improve the technology performance level (TPL) of wave energy converters at low technology readiness levels (TRLs), potentially reducing development costs incurred for excessive device refinement and redesign during prototyping, physical tank experiments and open ocean tests. A case study is presented, where the HiFiWEC is assessed by comparing its performance against lower fidelity wave-towire models, from which the following conclusions can be drawn:

- Hydrodynamic models based on boundary element method solvers, even when linear models are extended to include nonlinear FroudeKrylov forces and viscous effects, are unable to achieve accuracy levels provided by CFD-based approaches. This, along with the uncertainty in identifying the drag coefficient in the nonlinear viscous model, reinforces the need for CFD-based approaches in applications where high-fidelity is required.

- Excessive simplification of the power take-off model, neglecting aspects such as losses or constraints, can lead to significantly overestimated generated energy estimations. In fact, it is demonstrated that simplification in the power take-off model may result in considerably larger inaccuracies than simplification of the hydrodynamic model. Therefore, including a high-fidelity power take-off model is vital to accurately estimate the energy generation of a wave energy converter.

- Most importantly, the holistic performance of a wave energy converter can only be evaluated in high-fidelity by means of a comprehensive wave-to-wire simulation platform where both high-fidelity hydrodynamic and power take-off models are adequately coupled, since minor inaccuracies in either of this major stages of the wave-to-wire model can result in significant inaccuracy in generated power estimation.

\section{Acknowledgment}

This material is based upon works supported by the Science Foundation Ireland under Grant No. 13/IA/1886.

\section{Appendix A. BEM-based hydrodynamic model}

Time-domain hydrodynamic models based on BEM models use, in general, Cummins equation [75] as follows,

$M \ddot{z}_{d}(t)=-K_{H} z_{d}+\int_{-\infty}^{\infty} K_{e x}(t-\tau) \eta(\tau) d \tau-\mu_{\infty} \ddot{z}_{d}(t)-\int_{-\infty}^{\infty} K_{r a d}(t-\tau) \dot{z}_{d}(\tau) d \tau+F_{P T O}$

where $z_{d}, \dot{z}_{d}$ and $\ddot{z}_{d}$ are the displacement, velocity and acceleration of the WEC, respectively, $M$ is the mass of the WEC, $K_{H}$ the hydrostatic stiffness, $K_{e x}(t)$ the excitation impulse response function (IRF), $\mu_{\infty}$ the added-mass at infinite frequency, and $K_{\text {rad }}(t)$ the radiation IRF.

The linear hydrodynamic model, used in the $L B E M W 2 W$ and $L B E M+i P T O$ models, can be extended to include nonlinear effects such as nonlinear FK forces or viscous effects, as in the $N L B E M W 2 W_{C d=1}$ and $N L B E M W 2 W_{C d=2}$ models. Nonlinear FK forces can be included using the computationally efficient algebraic solution [76] as follows,

$M \ddot{z}_{d}(t)=F_{F K}(t)+\int_{-\infty}^{\infty} K_{\text {diff }}(t-\tau) \eta(\tau) d \tau-\mu_{\infty} \ddot{z}_{d}(t)-\int_{-\infty}^{\infty} K_{\text {rad }}(t-\tau) \dot{z}_{d}(\tau) d \tau+F_{P T O}+F_{v i s c}$

where $K_{\text {diff }}(t)$ is the diffraction IRF, and

$F_{F K}=F_{g}-\int_{0}^{2 \pi} \int_{\sigma_{1}}^{\sigma_{2}} P(x(\sigma, \theta), z(\sigma, \theta), t) f^{\prime}(\sigma) f(\sigma) d \sigma d \theta$. 
$F_{g}$ is the gravity force, the integration limits $\sigma_{1}=z_{d}-H$ and $\sigma_{2}=\eta$ define the instantaneous wetted surface, $H$ is the draft of the device, $\sigma$ and $\theta$ are the parametric cylindrical coordinates of the algebraic solution, and $x$ and $z$ the Cartesian coordinates.

Finally, viscous effects can be incorporated by using a Morison-like equation [77],

$F_{v i s c}=-\frac{1}{2} \rho C_{d} A_{d}(t)\left|\dot{z}_{d}-\dot{\eta}\right|\left(\dot{z}_{d}-\dot{\eta}\right)$

where $\rho$ is the density of water, $C_{d}$ the drag coefficient, $A_{d}$ the instantaneous cross-sectional area of the device, and $\dot{\eta}$ the velocity of the undisturbed water particles.

\section{References}

[1] REN21, Renewables 2017 global status report, Tech. rep. Paris: REN21 Secretariatl 2017

[2] Mork G, Barstow S, Kabuth A, Pontes MT. Assessing the global wave energy potential, In: ASME 29th international conference on ocean, offshore and arctic engineering, Shangai, China, vol. 3; 2010. p. 447-54.

[3] Edenhofer O, Pichs-Madruga R, Sokona Y, Seyboth K, Eickemeier P, Matschoss P, et al. IPCC special report on renewable energy sources and climate change mitigation, intergovernmental panel on climate change; 2011.

[4] Falcão AdO. Wave energy utilization: a review of the technologies. Renew Sustain Energy Rev 2010;14(3):899-918.

[5] Weber J. Wec technology readiness and performance matrix-finding the best research technology development trajectory. In: 4th International conference on ocean energy (ICOE). Dublin (Ireland); 2012.

[6] Penalba M, Ringwood JV. A review of wave-to-wire models for wave energy converters. Energies 2016;9(7):506.

[7] Ransley E, Greaves D, Raby A, Simmonds D, Hann M. Survivability of wave energy converters using CFD. Renew Energy 2017;109:235-47.

[8] Ransley E, Greaves D, Raby A, Simmonds D, Jakobsen M, Kramer M. RANS-VOF modelling of the wavestar point absorber. Renew Energy 2017;109:49-65.

[9] Schmitt P, Elsaesser B. On the use of OpenFOAM to model oscillating wave surge converters. Ocean Eng 2015;108:98-104.

[10] Schmitt P, Elsässer B. The application of froude scaling to model tests of oscillating wave surge converters. Ocean Eng 2017;141:108-15.

[11] Iturrioz A, Guanche R, Lara J, Vidal C, Losada I. Validation of OpenFOAM ${ }^{\circledR}$ for oscillating water column three-dimensional modeling. Ocean Eng 2015;107:222-36.

[12] Elhanafi A, Fleming A, Macfarlane G, Leong Z. Underwater geometrical impact on the hydrodynamic performance of an offshore oscillating water column-wave energy converter. Renew Energy 2017;105:209-31.

[13] Windt C, Davidson J, Ringwood J. High-fidelity numerical modelling of ocean wave energy systems: a review of computational fluid dynamics-based numerical wave tanks. Renew Sustain Energy Rev [in press].

[14] Amundarain M, Alberdi M, Garrido AJ, Garrido I. Modeling and simulation of wave energy generation plants: output power control. IEEE Trans Indust Electron 2011;58(1):105-17.

[15] Garrido AJ, Otaola E, Garrido I, Lekube J, Maseda FJ, Liria P, et al. Mathematical modeling of oscillating water columns wave-structure interaction in ocean energy plants. Math Prob Eng 2015;727982.

[16] Henriques J, Gomes R, Gato L, Falcão A, Robles E, Ceballos S, et al. Testing and control of a power take-off system for an oscillating-water-column wave energy converter. Renew Energy 2016;85(Supplement C):714-24.

[17] Bailey H, Robertson BR, Buckham BJ. Wave-to-wire simulation of a floating oscil lating water column wave energy converter. Ocean Eng 2016;125:248-60.

[18] Kelly JF, Wright WMD, Sheng W, OSullivan K. Implementation and verification of a wave-to-wire model of an oscillating water column with impulse turbine. IEEE Trans Sustain Energy 2016;7(2):546-53.

[19] Henderson R. Design, simulation, and testing of a novel hydraulic power take-off system for the pelamis wave energy converter. Renew Energy 2006;31(2):271-83.

[20] Josset C, Babarit A, Clément AH. A wave-to-wire model of the Searev wave energy converter. Proc Inst Mech Engin, Part M: J Eng Marit Environ 2007;221(2):81-93.

[21] Hansen RH, Kramer MM, Vidal E. Discrete displacement hydraulic power take-off system for the wavestar wave energy converter. Energies 2013;6(8):4001-44.

[22] Bailey H, Ortiz JP, Robertson B, Buckham BJ, Nicoll RS. A methodology for waveto-wire WEC simulations. In: Proceedings of the 2nd marine energy technology symposium. Seattle (WA); 2014.

[23] Garcia-Rosa PB, Vilela Soares Cunha JP, Lizarralde F, Estefen SF, Machado IR, Watanabe EH. Wave-to-wire model and energy storage analysis of an ocean wave energy hyperbaric converter. IEEE J Ocean Eng 2014;39(2):386-97.

[24] Kamizuru Y. Development of hydrostatic drive trains for wave energy converters, Ph.D. thesis; RWTH Aachen University; 2014.

[25] Cargo C, Hillis A, Plummer A. Strategies for active tuning of wave energy converter hydraulic power take-off mechanisms. Renew Energy 2016;94:32-47.

[26] Gaspar JF, Kamarlouei M, Sinha A, Xu H, Calvário M, Faÿ FX, et al. Speed control of oil-hydraulic power take-off system for oscillating body type wave energy converters. Renew Energy 2016;97:769-83.

[27] Tedeschi E, Carraro M, Molinas M, Mattavelli P. Effect of control strategies and power take-off efficiency on the power capture from sea waves. IEEE Trans Energy Convers 2011;26(4):1088-98.

[28] Sjolte J, Sandvik CM, Tedeschi E, Molinas M. Exploring the potential for increased production from the wave energy converter lifesaver by reactive control. Energies
$2013 ; 6(8): 3706-33$

[29] Polinder H, Damen ME, Gardner F. Linear pm generator system for wave energy conversion in the AWS. IEEE Trans Energy Convers 2004;19(3):583-9.

[30] Wu F, Zhang X-P, Ju P, Sterling MJ. Modeling and control of AWS-based wave energy conversion system integrated into power grid. IEEE Trans Power Syst 2008;23(3):1196-204

[31] O'Sullivan AC, Lightbody G. Co-design of a wave energy converter using constrained predictive control. Renew Energy 2017;102(Part A):142-56.

[32] Penalba M, Ringwood J. A high-fidelity wave-to-wire model for wave energy converters. Renew Energy [submitted for publication].

[33] Penalba M, Kelly T, Ringwood JV. Using NEMOH for modelling wave energy converters: a comparative study with WAMIT. In: Proceedings of the 12th European wave and tidal energy conference (EWTEC), vol. 631. Cork (Ireland); 2017.

[34] Giorgi G, Penalba M, Ringwood JV. Nonlinear hydrodynamic models for heaving buoy wave energy converters. In: Proceedings of the 3rd Asian wave and tidal energy conference; 2016. p. 144-53.

[35] Giorgi G, Ringwood JV. Consistency of viscous drag identification tests for wave energy applications. In: Proceedings of the 12th European wave and tidal energy conference (EWTEC), vol. 643. Cork (Ireland); 2017

[36] Davidson J, Giorgi S, Ringwood JV. Linear parametric hydrodynamic models for ocean wave energy converters identified from numerical wave tank experiments. Ocean Eng 2015;103:31-9.

[37] Giorgi S, Davidson J, Ringwood JV. Identification of wave energy device models from numerical wave tank data - part 2: data-based model determination. IEEE Trans Sustain Energy 2016;7(3):1020-7.

[38] Davidson J, Genest R, Ringwood JV. Adaptive control of a wave energy converter simulated in a numerical wave tank. In: Proceedings of the 12th European wave and tidal energy conference (EWTEC), vol. 747. Cork (Ireland); 2017.

[39] Davidson J, Windt C, Giorgi G, Genest R, Ringwood J. In: OpenFOAM: selected papers from the 11th workshop. Springer; 2018 [Ch. Evaluation of energy maximising control systems for wave energy converters using OpenFOAM].

[40] Lamont-Kane P, Folley M, Whittaker T. Investigating uncertainties in physical testing of wave energy converter arrays. In: Proceedings of the 10th European wave and tidal energy conference (EWTEC). Aalborg (Denmark); 2013.

[41] Draycott S, Noble D, Davey T, Steynor J, Ingram DM. Application of complex wave and current conditions in a laboratory environment. In: Proceedings of the 12th European wave and tidal energy conference (EWTEC), vol. 890. Cork (Ireland); 2017.

[42] Beatty S, Buckham B, Wild P. Experimental comparison of self-reacting point absorber WEC designs. In: Proceedings of the 10th European wave and tidal con ference (EWTEC), vol. 770. Aalborg (Denmark); 2013.

[43] Beatty S, Ferri F, Bocking B, Kofoed J, Buckham B. Power take-off simulation for scale model testing of wave energy converters. Energies 2017;10(7):973.

[44] Pedersen HC, Hansen RH, Hansen AH, Andersen TO, Bech MM. Design of full scale wave simulator for testing power take off systems for wave energy converters. Int J Mar Energy 2016;13:130-56.

[45] Tanizawa K. The state of the art on numerical wave tank. In: Proceedings of 4th Osaka colloquium on seakeeping performance of ships; 2000. p. 95-114.

[46] Mingham C, Qian L, Causon D. Numerical modeling of wave energy converter: state-of-the-art techniques for single WEC and converter arrays. Elsevier; 2016 [Ch. Computational Fluid Dynamics (CFD) Models, pp. 105-122].

[47] Weller HG, Tabor G, Jasak H, Fureby C. A tensorial approach to computational continuum mechanics using object-oriented techniques. Comp Phys 1998;12(6):620-31.

[48] Holzmann T. Mathematics, numerics, derivations and OpenFOAM, 4th ed.; 2016 [Ch. 11: SIMPLE, PISO and PIMPLE algorithm, pp. 111-134].

[49] Devolder B, Schmitt P, Rauwoens O, Elsaesser B, Troch P. A review of the implicit motion solver algorithm in OpenFOAM to simulate a heaving buoy. In: Proceedings of the 18th numerical towing tank symposium. Marstrand (Sweden); 2015.

[50] Windt C, Davidson J, Schmitt P, Ringwood JV. Assessment of numerical wave makers. In: Proceedings of the 12th European wave and tidal energy conference (EWTEC), vol. 707. Cork (Ireland); 2017.

[51] Jacobsen N, Fuhrmann DR, Fredsoe J. A wave generation toolbox for the opensource CFD library: OpenFoam(R). Int J Numer Meth Fluids 2012;70(9):1073-88.

[52] Davidson J, Cathelain M, Guillemet L, Le Huec T, Ringwood J. Implementation of an openfoam numerical wave tank for wave energy experiments. In: Proceedings of the 11th European wave and tidal energy conference (EWTEC), no. 09B1-1. Nantes (France); 2015

[53] Genest R, Davidson J, Ringwood JV. Adaptive control of a wave energy converter. IEEE Trans Sustain Energy 2018(99):1.

[54] Windt C, Davidson J, Benazzou A, Ringwood J. Performance assessment of the overset grid method for numerical wave tank experiments in the openfoam environment. In: Proceedings of the ASME 2018 37th international conference on 
ocean, offshore and arctic engineering, no. OMAE2018-77564; 2018.

[55] Penalba M, Sell N, Hillis A, Ringwood J. Validating a wave-to-wire model for wave energy converter - part I: the hydraulic transmission system. Energies 2017;10(7):977

[56] Jelali M, Kroll A. Hydraulic servo-systems: modelling, identification and control. Springer Science \& Business Media; 2012.

[57] Schlösser W. Mathematical model for displacement pumps and motors. Hydraul Power Transm 1961:252-7.

[58] Schlösser W. The overall efficiency of positive-displacement pumps. In: BHRA fluid power symposium; 1968. p. 34-48.

[59] Penalba M, Cortajarena J-A, Ringwood JV. Validating a wave-to-wire model for awave energy converter - part II: the electrical system. Energies 2017;10(7):1002.

[60] Krause PC, Wasynczuk O, Sudhoff SD, Pekarek S. Analysis of electric machinery and drive systems, third ed. IEEE Press Series on Power Engineering, Wiley-Blackwell; 2013.

[61] Palm J, Eskilsson C, Paredes GM, Bergdahl L. Cfd simulation of a moored floating wave energy converter. In: Proceedings of the 10th European wave and tidal energy conference (EWTEC), vol. 554. Aalborg (Denmark); 2013.

[62] Penalba M, Mérigaud A, Gilloteaux J-C, Ringwood JV. Influence of nonlinear Froude-Krylov forces on the performance of two wave energy points absorbers. J Ocean Eng Mar Energy 2017;3(3):209-20.

[63] Penalba M, Ringwood J. The impact of wave-to-wire models in control parameter optimisation and power assessment. In: ASME. Proceedings of the 37th international conference on ocean, offshore and arctic engineering, OMAE, no. OMAE2018-77501; 2018.

[64] Penalba M, Ringwood J. Linearisation-based nonlinearity measures for wave-towire models in wave energy. Ocean Eng [submitted for publication].

[65] Penalba M, Touzón I, Lopez-Mendia J, Nava V. A numerical study on the hydrodynamic impact of device slenderness and array size in wave energy farms in realistic wave climates. Ocean Eng 2017;142:224-32.
[66] Hasselmann K, Barnett T, Bouws E, Carlson H, Cartwright D, Enke K, et al. Measurements of wind-wave growth and swell decay during the joint north sea wave project (JONSWAP), Tech. rep. Hamburg: Deutsches Hydrographisches Institut; 1973.

[67] Windt C, Davidson J, Schmitt P, Ringwood J. A benchmark study for numerical wavemakers in CFD simulations. Coast Eng [submitted for publication].

[68] Schmitt P, Danisch L, Lamont-Kane P, Elsäßer B. Designing short-term wave traces to assess wave power devices. J Offshore Polar Eng 2018;28:46-53.

[69] Hansen RH, Andersen TO, Pedersen HC. Model based design of efficient power takeoff systems for wave energy converters. In: Proceedings of the 12th Scandinavian international conference on fluids power.

[70] Bacelli G, Ringwood JV, Gilloteaux J-C. A control system for a self-reacting point absorber wave energy converter subject to constraints. IFAC Proc Vol 2011;44(1):11387-92

[71] Genest R, Ringwood JV. A critical comparison of model-predictive and pseudospectral control for wave energy devices. J Ocean Eng Mar Energy 2016;2(4):485-99.

[72] Faedo N, Olaya S, Ringwood JV. Optimal control, MPC and MPC-like algorithms for wave energy systems: an overview. IFAC J Syst Control 2017;1:37-56.

[73] Cathey JJ, Cavin RK, Ayoub A. Transient load model of an induction motor. IEEE Trans Power Appar Syst 1973(4):1399-406.

[74] Davidson J, Ringwood JV. Mathematical modelling of mooring systems for wave energy converters: a review. Energies 2017;10(5):666.

[75] Cummins WE. The impulse response function and ship motions, Tech. rep. DTIC Document; 1962.

[76] Giorgi G, Ringwood JV. Computationally efficient nonlinear Froude-Krylov force calculations for heaving axisymmetric wave energy point absorbers. J Ocean Eng Mar Energy 2017;3(1):21-33.

[77] Morison JR, O'Brien MP, Johnson JW, Schaaf SA. The forces exerted by surface waves on piles. AIME Petrol Trans 1950;189:149-57. 